Accepted by ApJ on June 21, Published Aug 20, 2013

\title{
Diffraction-limited Visible Light Images of Orion Trapezium Cluster With the Magellan Adaptive Secondary AO System $(\mathrm{MagAO})^{1}$
}

\author{
Close, L.M. ${ }^{1}$, Males, J.R. ${ }^{1}$, Morzinski, K. ${ }^{1,4}$, Kopon, D. ${ }^{1,5}$, Follette, K. ${ }^{1}$, Rodigas, T. J. ${ }^{1}$, \\ Hinz, P. ${ }^{1}$, Wu, Y-L. ${ }^{1}$, Puglisi, A. ${ }^{2}$, Esposito, S. ${ }^{2}$, Riccardi, A. ${ }^{2}$, Pinna, E. ${ }^{2}$, Xompero, M. ${ }^{2}$, \\ Briguglio, R. ${ }^{2}$, Uomoto, A, ${ }^{3}$, Hare, T. ${ }^{3}$ \\ lclose@as.arizona.edu \\ ${ }^{1}$ Steward Observatory, University of Arizona, Tucson, AZ 85721, USA \\ ${ }^{2}$ INAF - Osservatorio Astrofisico di Arcetri, I-50125, Firenze, Italy \\ ${ }^{3}$ Carnegie Observatories, 813 Santa Barbara Street, Pasadena, California, 91101 USA \\ ${ }^{4}$ NASA Sagan Fellow \\ ${ }^{5} \mathrm{MPiA}$, Germany
}

\begin{abstract}
We utilized the new high-order (250-378 mode) Magellan Adaptive Optics system (MagAO) to obtain very high spatial resolution observations in "visible light" with MagAO's VisAO CCD camera. In the good-median seeing conditions of Magellan $\left(0.5-0.7^{\prime \prime}\right)$ we find MagAO delivers individual short exposure images as good as 19 mas optical resolution. Due to telescope vibrations, long exposure $(60 \mathrm{~s})$ r' $(0.63 \mu \mathrm{m})$ images are slightly coarser at FWHM=23-29 mas (Strehl $\sim 28 \%$ ) with bright $(R<9 \mathrm{mag})$ guide stars. These are the highest resolution filled-aperture images published to date. Images of the young $(\sim 1$ Myr) Orion Trapezium $\theta^{1}$ Ori A, B, and C cluster members were obtained with VisAO. In particular, the 32 mas binary $\theta^{1}$ Ori $C_{1} C_{2}$ was easily resolved in non-interferometric images for the first time. Relative positions of the bright trapezium binary stars were measured with $\sim 0.6-5$ mas accuracy. We now
\end{abstract}

\footnotetext{
${ }^{01}$ This paper includes data gathered with the 6.5 meter Magellan Telescopes located at Las Campanas Observatory, Chile.
} 
are sensitive to relative proper motions of just $\sim 0.2 \mathrm{mas} / \mathrm{yr}(\sim 0.4 \mathrm{~km} / \mathrm{s}$ at 414 pc) - this is a $\sim 2-10 \times$ improvement in orbital velocity accuracy compared to previous efforts. For the first time, we see clear motion of the barycenter of $\theta^{1}$ Ori $B_{2} B_{3}$ about $\theta^{1}$ Ori $B_{1}$. All five members of the $\theta^{1}$ Ori $B$ system appear likely a gravitationally bound "mini-cluster", but we find that not all the orbits can be both circular and co-planar. The lowest mass member of the $\theta^{1}$ Ori $B$ system $\left(B_{4}\right.$; mass $\left.\sim 0.2 M_{\odot}\right)$ has a very clearly detected motion (at $4.1 \pm 1.3 \mathrm{~km} / \mathrm{s}$; correlation $=99.9 \%)$ w.r.t $B_{1}$. Previous work has suggested that $B_{4}$ and $B_{3}$ are on long-term unstable orbits and will be ejected from this "mini-cluster". However, our new "baseline" model of the $\theta^{1}$ Ori $B$ system suggests a more hierarchical system than previously thought, and so the ejection of $B_{4}$ may not occur for many orbits, and $B_{3}$ may be stable against ejection long-term. This "ejection" process of the lowest mass member of a "mini-cluster" could play a major role in the formation of low mass stars and brown dwarfs.

Subject headings: instrumentation: adaptive optics — binaries: general — stars: evolution — stars: formation — stars: low-mass, brown dwarfs

\section{INTRODUCTION}

\subsection{The Need For High-Resolution Imaging}

It is critical to the understanding of the motions and masses of stars, brown dwarfs, and exoplanets to obtain the highest resolution images possible. In fact, almost every aspect of astronomical science benefits from the highest spatial resolutions possible. The highest resolution "maps" at the milliarcsec (mas) resolution scales $\left(0.001^{\prime \prime}=1\right.$ mas) are being produced by interferometry (like VLTI/AMBER in the IR). However, interferometric techniques suffer from incomplete uv coverage and object models are usually required to interpret interferometric data. Moreover, combining multiple $8 \mathrm{~m}$ telescopes together in the VLTI and waiting for the Earth's rotation to fill in the uv plane is both time consuming and expensive (hence limiting the general utility of large surveys with VLTI, for example).

Imaging from space with a filled aperture (and so complete uv coverage) with HST has proven to be very productive, but HST's small $2.4 \mathrm{~m}$ aperture, combined with a need for large pixels, limits its best spatial resolutions to 50-100 mas. Also HST is considerably more expensive than any other telescope and its lifetime is limited. Large $(8-10 \mathrm{~m})$ ground-based telescopes can match HST's $\sim 50$ mas V-band resolution with adaptive optics in the NIR $(1.2-2.4 \mu \mathrm{m})$. For example, the 8.4m LBTAO system (FLAO; Esposito et al. (2011)) can also 
achieve deep 50 mas resolution images with AO at $1.64 \mu m$ (Close et al. 2012b). However, to achieve deep images better than $\sim 40-50$ mas is impossible with the current generation of facility AO systems and cameras. For example, to reach $\leq 20$ mas resolutions at $\mathrm{H}$ band $(1.65 \mu \mathrm{m})$ would require a $\mathrm{D} \geq 16 \mathrm{~m}$ filled-aperture telescope. Hence it will not be until the ELT era (early, to mid, 2020s) that images in the NIR will be significantly sharper than 20 mas.

\subsection{Into the Blue: Adaptive Optics in the Visible}

However, there is another approach to reaching these resolutions. While $8-10 \mathrm{~m} \mathrm{AO}$ performance is limited to $\geq 40$ mas in the NIR, it is possible to gain a factor of two improvement in resolution by moving to shorter (bluer) wavelengths for AO correction. This so called "visible AO" can theoretically reach 16 mas resolutions on an $8 \mathrm{~m}$ telescope at $0.656 \mu \mathrm{m}\left(H_{\alpha}\right)$. However, the complexity of an 8-10m class AO system designed for optical wavelengths ( $>500$ modes, at $>1 \mathrm{KHz}$ ) is beyond that of the current facility systems (with perhaps the exception of the FLAO system on the $8.4 \mathrm{~m}$ LBT which; however, currently has no facility visible AO CCD science camera (Esposito et al. 2010b)).

We note that AO with "lucky" imaging in the visible has been successfully used at the somewhat smaller 5m Palomar (Law et al. 2009) and has reached resolutions of 35 mas, recently the Palm3000 system has demonstrated excellent corrections (Dekany et al. 2013). Improved Lucky visible imaging (image synthesis based on Fourier Amplitude selection) has

also been developed by Garrel, Guvon, \& Baudoz (2012). Visible AO has been done before on much smaller telescopes like Robo-AO on the $1.5 \mathrm{~m}$ at Palomar (Barnec et al. 2012) or the Villages project on the 1.0m Nickel at Lick (Morzinski et al. 2010). In the near future some polarization work will be done in the visible with the $8 \mathrm{~m}$ VLT with the SPHERE AO system and ZIMPOL (Bazzon et al. 2012). Yet, MagAO is the first large $(D \geq 6.5 \mathrm{~m})$ telescope AO system designed to work in the visible -complete with a facility CCD AO science camera (VisAO). The MagAO commissioning results presented here inform us on the utility of large telescope visible AO performance.

\subsection{The Magellan AO System}

We have developed an AO system (inspired, in large part, by LBT's FLAO system; Esposito et al. (2012)) that can reach 20 mas resolutions with just 250-378 modes at $1 \mathrm{kHz}$ sampling speeds. It is important that such a visible AO system be located at an excellent 
site where the median seeing is less than $0.64^{\prime \prime}$. To achieve an AO fitting error small enough to reach $110 \mathrm{~nm} \mathrm{rms}$ total wavefront error (WFE) with 250 modes requires a telescope diameter of $\mathrm{D} \leq 6.5 \mathrm{~m}$. Hence, a solution to this design problem is a fast $(<1 \mathrm{~ms}$ response time) 585 element second generation adaptive secondary mirror (ASM) with a $1 \mathrm{kHz}$ Pyramid wavefront sensor (PWFS). These are exactly the characteristics of the Magellan Adaptive Secondary AO system (MagAO) deployed on the $0.64^{\prime \prime}$ median seeing (Thomas-Osip et al. 2010) 6.5m Magellan telescope at Las Campanas Observatory, Chile.

MagAO, with its VisAO camera 2 , is the first large telescope $(\geq 6.5 \mathrm{~m})$ facility AO system deployed that is targeting science in the visible $(0.6-1.1 \mu \mathrm{m})$. As will be shown in later in this paper, MagAO at first light produced long exposure (60s) diffraction-limited (110 nm WFE; $28 \%$ Strehl) $0.63 \mu \mathrm{m}$ images. Note during the first light run (commissioning run \#1; Nov/Dec 2013) we were limited to 250 corrected modes 3 . For more technical details about MagAO itself please see Close et al. (2012a).

It is important to note that MagAO sends all the infrared light into the Clio2 NIR (1-5.3 $\mu \mathrm{m}$ ) camera (Hinz et al. 2010; Morzinski et al. 2013)), whereas the visible light $(\lambda<1.1 \mu m)$ is split by a selectable beamsplitter between the PWFS and the VisAO (0.6-1.1 $\mu \mathrm{m})$ science camera (for more on the VisAO camera see Males et al. (2012); Kopon et al. (2012); Close et al. (2012a)). Hence all three focal stations (Clio2, VisAO, and PWFS) work simultaneously on all targets, allowing Visible and IR science to be done simultaneously.

\subsection{First Light VisAO Science: Motions of the Massive Young Stars in The Orion Trapezium Cluster}

Clearly the exciting possibility of obtaining $\sim 20$ mas FWHM images with MagAO could enhance our understanding of the positions (and motions) of the nearest massive young stars. Hence we targeted the Orion Trapezium cluster during the first light commissioning run with the MagAO system.

The study of the motions of the young stars in the Trapezium cluster is an important problem (see for example McCarthy \& Zuckerman (2004); Close et al. (2012b); Grellmann et al.

\footnotetext{
${ }^{2}$ see http://visao.as.arizona.edu/for web resources, SPIE publications, and guides for observers

${ }^{3}$ During the recent commissioning run \#2 (March/April 2013) MagAO had a better modal basis set which allowed on-sky closed-loop stability at its maximum of 378 corrected modes. Hence MagAO currently can achieve a WFE of just $102 \mathrm{~nm}$ rms with 378 modes in $0.5^{\prime \prime}$ seeing on bright stars $(R \leq 9$ mag). However, this paper concerns the first light (commissioning run \#1) results of MagAO where only 250 modes were corrected.
} 
(2013)). After all, the detailed formation of stars is still a poorly understood process. In particular, the formation mechanism of the lowest mass stars and brown dwarfs is uncertain. Detailed 3D (and N-body) simulations of star formation by Bate et al. (2002, 2003, 2009, 2011) and Parker et al. (2011) all suggest that stellar embryos frequently form into "miniclusters" which dynamically decay, "ejecting" the lowest mass members. Such theories can explain why there are far more field brown dwarfs (BD) compared to BD companions of solar type stars (McCarthy \& Zuckerman 2004) or early M stars (Hinz et al. 2002). Moreover, these theories which invoke some sort of dynamical decay (Durisen. Sterzik, \& Pickett 2001) or ejection (Reipurth \& Clarke 2001) suggest that there should be no wide (>20 AU) very low mass (VLM; $M_{t o t}<0.185 M_{\odot}$ ) binary systems observed in the field (age $\sim 5$ Gyr). Indeed, the AO surveys of Close et al. (2003a) and the HST surveys of Reid et al. (2001a); Burgasser et al. (2003); Bouv et al. (2003); Gizis et al. (2003) have not discovered more then a few wide ( $>16 \mathrm{AU}$ ) VLM systems in the field population (for a review see Burgasser et al. (2007)). Additionally, the dynamical biasing towards the ejection of the lowest mass members naturally suggests that the frequency of field VLM binaries should be much lower $(\lesssim 5 \%$ for $\left.M_{t o t} \sim 0.16 M_{\odot}\right)$ than for more massive binaries $\left(\sim 60 \%\right.$ for $\left.M_{t o t} \sim 1 M_{\odot}\right)$. Indeed, observations suggest that the binarity of VLM systems with $M_{t o t} \lesssim 0.185 M_{\odot}$ is $10-15 \%$ (Close et al. 2003a; Burgasser et al. 2003, 2007) which, although higher than predicted is still lower than that of the $\sim 42 \%$ of more massive M-dwarfs Fischer \& Marcv (1992) or $\sim 60 \%$ of G star binaries Duquennoy \& Mayor (1991). However as is noted in Close et al. (2007) there is evidence that in young clusters wide VLM binaries are much more common than in the old field population. They attribute this to observing these wide VLM systems before they are destroyed by encounters in their natal clusters. Hence, we need to look at nearby young clusters to see these low-mass objects in "mini-clusters" (of a few bound stars) before ejection has occurred.

Despite the success of these decay, or ejection, scenarios in predicting the observed properties of low mass VLM stars and binaries, it is still not clear that "mini-clusters" even exist in the early stages of star formation. To better understand whether such "miniclusters" do exist we have examined the closest major OB star formation cluster for signs of such "mini-clusters". Here we focus on the $\theta^{1}$ Ori stars in the famous Orion Trapezium cluster. Trying to determine if some of the tight star groups in the Trapezium cluster are gravitationally bound is a first step to determining if bound "mini-clusters" exist. Also it is important to understand the true number of real, physical, binaries in this cluster, as there is evidence that the overall number of binaries is lower (at least for the lower mass members) in the dense trapezium cluster compared to the lower density young associations like Taurus-Auriga (McCaughrean 2000; Kohler et al. 2006). In particular, we will examine the case of the $\theta^{1}$ Ori $\mathrm{A}, \mathrm{B}$ and $\mathrm{C}$ groups in detail. 
The Trapezium OB stars $\left(\theta^{1}\right.$ Ori A, B, C, D, and E; see Fig. 1) consists of the most massive OB stars located at the center of the Orion Nebula star formation cluster (for a review see Genzel \& Stutzki (1989)). Due to the nearby (VLBA trigonometric parallax distance of $414 \pm 7$ pc; Menten et al. (2007)), and luminous nature of these stars they are a unique laboratory for the study of a high-mass star formation cluster (the dominate birthplace for stars of all masses), and have been the target of several high-resolution imaging studies. For brevity, we do not reproduce here a complete history of past high resolution surveys of Trapezium, please see Close et al. (2012b) instead for a review.

Close et al. (2012b) utilized the LBT FLAO system to map out the Trapezium in narrow-band NIR filters at $\sim 50-60$ mas resolutions. It total Close et al. (2012b) analyzed 14 years of observations of the cluster. Yet, only the LBT 2011 observations were of very high quality. In this paper we present the first high-resolution visible $(0.57-0.68 \mu \mathrm{m})$ AO images. These images are of the Trapezium cluster and reach very high resolutions of $\sim 23$ mas. We have now over 15 years of observations of this field with at $<0.08^{\prime \prime}$ resolution. More importantly, we now have two complete high-quality datasets from LBT and MagAO, that track motion of the Trapezium stars at $<0.05^{\prime \prime}$ resolutions.

In this paper we outline how these MagAO observations were carried out with the new VisAO camera. We detail how these data were calibrated and reduced and how the stellar positions were measured. We resolve the 32 mas binary $\theta^{1}$ Ori $\mathrm{C}$ in a filled aperture image for the first time. We compare the measured astrometry for $\theta^{1}$ Ori $\mathrm{C} 1$ and $\mathrm{C} 2$ against its published (interferometric) orbit. We also fit the observed positions to calculate velocities (or upper limits) for the $\theta^{1}$ Ori $B_{1}, B_{2}, B_{3}, B_{4} \& A_{1}, A_{2}$ stars. While Schertl et al. (2003) and Close et al. (2003c, 2012b) had hints that the $\theta^{1}$ Ori B group may be a bound "mini-cluster" -we here show it is clearly so, with the first detection of curvature in the orbital motion of members of this group. We also present the first model for how the complex set of orbits in the $\theta^{1}$ Ori B mini-cluster could (and cannot) be arranged.

\section{INSTRUMENTAL SET-UP}

We utilized MagAO to obtain the first diffraction-limited (and unsaturated) images of the young stars in the Trapezium cluster in the visible $(0.6-0.7 \mu m)$. This is not a simple task, since as telescopes have increased in size, bright stars tend to now saturate -even in the shortest possible exposures. Hence special precautions are needed to avoid saturation of the bright Trapezium stars themselves. It is difficult to make unsaturated, but diffractionlimited, "visible light" images of the bright Trapezium stars with modern $6.5 \mathrm{~m}$ class AO systems at even moderately high Strehl. Witness the fact that this is the first such dataset 
ever published. The following subsections outline how this was accomplished.

The MagAO system is unique (at least in the southern hemisphere) in many ways. To reduce the aberrations caused by atmospheric turbulence all large telescope AO systems have a deformable mirror which is updated in shape at $\sim 500 \mathrm{~Hz}$. Except for the MMTAO and LBTAO systems (Wildi et al. 2003a; Esposito et al. 2011) all other adaptive optics systems have located this deformable mirror (DM) at a re-imaged pupil (effectively a compressed image of the primary mirror). To reimage the pupil onto a DM typically requires 3-8 warm additional optical surfaces, which significantly increases the thermal background and decreases the optical throughput of the system (Lloyd-Hart 2000). However, MagAO utilizes a next generation adaptive secondary DM. This DM is both the secondary mirror of the telescope and the DM of the AO system. In this manner there are no additional optics required in front of the science camera. Hence the emissivity is lower, throughput higher. MagAO's DM is an advanced "second generation" adaptive secondary mirror (ASM; similar to those on the LBT), which enables the highest on-sky visible Strehl ( $>25 \%$ at r' band; 0.57-0.68 $\mu \mathrm{m}$ ) of any large $6.5-10 \mathrm{~m}$ telescope today.

The MagAO ASM consists of 585 voice coil actuators that push (or pull) on 585 small magnets glued to the backsurface of a thin $(1.6 \mathrm{~mm}), 850 \mathrm{~mm}$ aspheric ellipsoidal Zerodur glass "shell" (for a detailed review of the secondary mirror see Close et al. (2012a)). Like in the case of the LBT AO system we have complete positional control of the surface of this reflective shell by use of a $70 \mathrm{kHz}$ capacitive sensor feedback loop. This positional feedback loop allows one to position an actuator of the deformable shell to within $\sim 5 \mathrm{~nm} \mathrm{rms}$ (total residual polishing wavefront errors (mainly at inter actuator scales) amount to only $\sim 50$ $\mathrm{nm}$ rms over the whole secondary). The AO system samples (and drives the ASM) at 990 $\mathrm{Hz}$ using 250-378 active controlled modes (with 585 actuators) on bright stars $(\mathrm{R}<9 \mathrm{mag})$.4

The wavefront slopes are measured with a very accurate, well calibrated, low aliasing error, Pyramid Wavefront sensor (PWFS). This is the second large telescope to use a PWFS (after the LBT, Esposito et al. (2011)). The performance of the MagAO PWFS is excellent. The very low residual wavefront errors obtained by the PWFS + ASM combination is due, in part, to the very accurate (high $\mathrm{S} / \mathrm{N}$ ) interaction matrix that can be obtained in closed-loop daytime calibrations with a retro-reflecting calibration return optic (CRO; Kopon et al. (2012)) that takes advantage of the Gregorian (concave) nature of the sec-

\footnotetext{
${ }^{4}$ With the PWFS we can operate on fainter stars by increasing the binning of the CCD39 in the PWFS. Hence for fainter guide stars with $9<R<12.7$ mag bin $2 \times 2$ and 120 modes are used. Likewise for $14.2<R<15.6 \mathrm{mag}$ bin $3 \times 3$ and 66 modes, $14.2<R<15.6 \mathrm{mag}$ bin $4 \times 4$ and 28 modes, and for the faintest stars $(15.6<R<16.5 \mathrm{mag})$ then bin $5 \times 5$ and just 21 modes are corrected. Once we are fainter than $R>12.7 \mathrm{mag}$ visible AO correction is very poor and science can only be done in the NIR with Clio2.
} 
ondary. To guarantee strict "on-sky" compliance with the "daytime calibrated" interaction matrix pupil/ASM/PWFS geometry the PWFS utilizes a novel "closed-loop pupil alignment system" that maintains the pupil alignment to $<2.5 \mu m$ (at the PWFS CCD39 images of the 4 pupils produced by the PWFS) during all closed-loop operations on bright stars. Moreover, we use a fixed pupil mask on the ASM to maintain the exact same pupil illumination when the CRO is used and also when we are on sky - so that our interaction matrices are valid (on and off sky). For a detailed review of the MagAO system see Close et al. (2012a) and references within.

\subsection{The MagAO PSF and Calculating Strehl}

During the MagAO first light commissioning run we observed the $\theta^{1}$ Ori $\mathrm{A}, \mathrm{B}$ and $\mathrm{C}$ groups on the nights of Dec 3,4, and 82012 (UT). The AO system corrected the lowest 250 system modes and was updated at $990 \mathrm{~Hz}$. The PWFS pupil was close-loop stabilized and the shell was protected from wind with a windscreen at the secondary mirror. Cooling pumps (for Clio2, VisAO, and the PWFS) added some vibrational blurring into the PSF. After commissioning run 1 these pumps were much better isolated. Nevertheless, the PSFs were still close to perfectly diffraction-limited. To better gauge the effectiveness of the AO correction we need to be able to measure the long-exposure PSF and calculate the Strehl of the PSF.

On bright $(R<9)$ guide stars in $\sim 0.6^{\prime \prime}$ V-band seeing we could obtain deep 5 minute PSF images (with no SAA or post-detection processing) with Strehls of $43 \%$ at $Y_{\text {short }}$ (Ys; $0.98 \mu \mathrm{m}$ ), or $140 \mathrm{~nm} \mathrm{rm}$ wavefront error (by use of the extended Marechal's approximation; see Fig. 2). We note the deep 5 minute image in Fig. 2 suffered from some additional vibrational blurring due to the cooling pumps for the CCDs and Clio25. These deep PSF images helped model the PSF to calculate Strehls for MagAO on $\theta^{1}$ Ori C which was so bright that only a $64 \times 64 \mathrm{CCD}$ window could be readout without saturation on $C_{1}$. Hence the wings of the PSF (beyond the $64 \times 64$ window) had to be estimated from a wavelength scaled PSF "halo" model based on the measured deep PSF wings of Figure 2, In this manner realistic Strehls could be estimated reliably from the small $64 x 64$ images of $\theta^{1}$ Ori $C_{1} 6_{6}^{6} \mathrm{We}$ note that it was only the Strehl of $\theta^{1}$ Ori $C_{1}$ that required this bootstrap approach all other Strehls (from full frame CCD images) in this paper were measured in the usual manner by

\footnotetext{
${ }^{5}$ For the data collection of Trapezium images in this paper the vibrating cooling pumps were temporarily powered off to help stabilize the images and obtain WFE $\sim 110 \mathrm{~nm}$ rms. We note that during the second commissioning run the Clio2 pump was successfully removed from the moving telescope structure and the CCD pump was better isolated from the telescope, greatly reducing residual vibrations.
} 
comparison to our model theoretical PSF.

\subsection{The VisAO CCD AO Science Camera}

These observations utilized the first facility visible light AO science camera (VisAO; (Males et al. (2012); Kopon et al. (2012)). VisAO has a fast, frame transfer, 1024x1024 0.5$1.1 \mu \mathrm{m}$ E2V CCD47 detector. We used the 64x64 window mode to minimize saturation on the array while observing $\theta^{1}$ Ori $\mathrm{C} 1$ (V=5.13 mag). While the $\sim 10 \times$ fainter $\theta^{1}$ Ori B1 $(\mathrm{V}=7.2)$ allowed the whole CCD to be readout without saturation.

The VisAO focal plane platescales were calibrated by the astrometry of four stars in the HD 40887 quadruple system and $\theta^{1}$ Ori $B_{1}$ and $\theta^{1}$ Ori $E_{1} 7$ (see sections 3 and 4 for more details about how the images were first reduced).

The positions (found by the IRAF allstar PSF fitting task) of these stars from our VisAO images were compared to unsaturated astrometry from Close et al. (2012b) which itself is derived from the HST ACS astrometry of Ricci et al. (2007). VisAO platescales and rms errors were then determined for the $H_{\alpha}$, [OI], r', i', z' and Ys filters with the IRAF geomap task. The platescale found was $0.0078513 \pm 0.000015^{\prime \prime} /$ pix at $H_{\alpha}(0.656 \mu m)$, and [OI] $(0.63 \mu \mathrm{m})$ providing a $8.03 \times 8.03^{\prime \prime}$ FOV with our $\mathrm{f} / 52.5$ beam on the CCD47's 13.0 $\mu \mathrm{m}$ pixels. At r' $(0.63 \mu \mathrm{m})$ the platescale was slightly coarser at $0.007917 \pm 0.000015^{\prime \prime} / \mathrm{pix}$, at z' $(0.906 \mu m)$ just slightly finer at $0.007911^{\prime \prime} \pm 0.000012^{\prime \prime} /$ pix, and at $Y_{\text {short }}(0.982 \mu m)$ $0.007906^{\prime \prime} \pm 0.000014^{\prime \prime} /$ pix. By design, the $\mathrm{f} / 16$ beam (direct from the ASM) is slowed down to $\mathrm{f} / 52.5$ to yield these very fine 7.9 mas/pixel VisAO platescales. We note this is one of the finest platescales ever for a facility camera.

Small distortions were detected by dithering a binary across the VisAO CCD. In this manner we detected a small change in the $\mathrm{Y}$ platescale $(\leq 1 \%)$ from the top of the array to the bottom. The exact formula to correct a binary with a primary star at position $\mathrm{X}, \mathrm{Y}$ of separation $\delta x$ and $\delta y$ for any residual distortions is true $_{\delta x}=$ measured $_{\delta x}-$ $\delta d x /\left(\right.$ abs $_{\left.\left.\text {measured }_{\delta x}\right] / 110.0\right)}$ and true $_{\delta y}=$ measured $_{\delta y}-\delta d y /\left(\right.$ abs $_{\text {measured }}$ my $\left.] / 44.5\right)$ where $\delta d x=-0.00038921676 *(X-512)+0.00084322443 *(Y-512)$ and $\delta d y=-0.00025760395 *$

\footnotetext{
${ }^{6}$ Note that to accurately calculate the Strehl of the $\theta^{1}$ Ori $C_{1}$ PSF required simply subtracting the PSF of $\theta^{1}$ Ori $C_{2}$ with the IRAF daophot allstar task.

${ }^{7}$ Typically the stars in the Trapezium used for this platescale test move at only $\sim 0.0015^{\prime \prime} / \mathrm{yr}$ so the platescale error over the $6.24^{\prime \prime}$ distance is $\sim 2 x 10^{-4}$ error — which is much smaller than the magnitude $(\sim 0.1 \%)$ of the platescale errors $-\sim 0.06^{\prime \prime}$ over this distance.
} 
$(X-512)-0.0024045175 *(Y-512)$ our observations were near the center of the detector and so these corrections were actually very small $(0.1-0.5 \%$ or $0.1-5$ mas changes to the $0.1-1^{\prime \prime}$ binaries), nevertheless all binary observations in this paper have been fully distortion corrected.

To determine the orientation of the $\mathrm{Y}$ axis of the VisAO images (which were all taken with the rotator following) it was first necessary to rotate each image counterclockwise (with the IRAF rotate task) by the ROTOFF FITS keyword value +90 degrees. At this point it was found by geomap that the direction of North was slightly $\left(0.890^{\circ}\right)$ East of VisAO's Y axis compared to the HST ACS Ricci et al. (2007) and LBT images (Close et al. 2012b) of the field. Hence a final counterclockwise rotation of $-0.890^{\circ}$ was applied to the final image. The rms uncertainty adopted for the MagAO rotator angle is estimated as $\sim 0.3^{\circ}$ this is the maximum error seen between different images of these stars on different nights. We suspect that this value of $\sim 0.3^{\circ}$ is quite conservative based on the very low scatter in the PA fits shown later in this paper.

\section{OBSERVATIONS \& REDUCTIONS}

For the $\theta^{1}$ Ori $\mathrm{C}$ field we locked the AO system (at 990Hz, 250 modes) in $0.5-0.7^{\prime \prime}$ seeing on the bright O5pv binary star $\theta^{1}$ Ori $C(\mathrm{~V}=5.13 \mathrm{mag})$ and used a $64 \times 64$ window in the center of the VisAO CCD with a set of $2608 \times 0.023$ second (60s total) unsaturated exposures at $H_{\alpha},[\mathrm{OI}]$, and r'. Immediately following the unsaturated exposures a set of 60 second exposures were obtained with the AO off. We note that $\theta^{1}$ Ori $\mathrm{C}$ is really a $\sim 0.03$ " binary composed of $\mathrm{C} 1$ and $\mathrm{C} 2$, (see Kraus et al. (2007) for more details).

Then the AO system was locked (250 modes, $990 \mathrm{~Hz}$ ) on $\theta^{1}$ Ori B1 (V=7.96 mag) and VisAO was used over its fullframe $(1 k \times 1 k)$ pixels to produce a set of $212 \times 0.283 \mathrm{~s}$ unsaturated (60s total) images at z'.

The individual frames were reduced in a normal manner. We used our custom AO image reduction script of Close et al. (2003a) to sky/bias subtract, cross-correlate (when needed), and median combine each image. The final individual image sets of the $\mathrm{C}$ and $\mathrm{B}$ fields each had a total exposure time of $1 \mathrm{~min}$. Figure 1 is a large FOV LBT NIR AO image from Close et al. (2012b) that defines the nomenclature and relative positions of the Trapezium stars for clarity.

In figure 2 one can see the marked improvement in resolution ( $~ 600$ mas to 34 mas) and Strehl ( $\sim 0.5 \%$ to $43 \%$ ) having the AO loop closed makes to a 300 second exposure. We note these Ys images are not post-detection "frame selected" (lucky imaging) nor shift and 
added (SAA) - so they are true 300s open-shutter exposures.

In figure 3 we show typical images of the binary $\theta^{1}$ Ori $C_{1}$ and $C_{2}$ imaged in $0.5^{\prime \prime}$ seeing ([OI] and r') on Dec 8, 2012 and worse $0.7^{\prime \prime}$ seeing for $H_{\alpha}$ on Dec 3, 2012. In all cases excellent (26-29 mas and 28-25\% Strehl) images are obtained.

In the middle row of figure 3 we retrieve the true resolution of the optical beam on the CCD47 by post-detection alignment of the images ( $\sim 2-4$ mas improvement). We also make a similarly small resolution improvement $(\sim 2-4$ mas) by removing the blurring effects of the CCD47's pixel response function (PRF). The CCD47's 13.0 $\mu \mathrm{m}$ pixel PRF was calibrated by noting the slight improvement in FWHM when a lab CCD with smaller $5.5 \mu \mathrm{m}$ pixels were used (instead of the $13 \mu \mathrm{m}$ pixels) in a PRF lab test. A similar amount (just slightly less) $\mathrm{PRF}$ is observed with HST's ACS CCD 8 . Once vibrations and PRF are minimized the images have 21-23 mas resolutions.

Very short (23 msec) individual images were not effected as much by the residual vibrations and achieved very high resolutions of 21 mas (see Fig. 4). These vibrations were found in commissioning to be mainly residual $60 \mathrm{~Hz}$ vibrations not corrected by $\mathrm{MagAO}$ and are likely due to a few fans on the telescope that we could not turn off. However, once corrected for PRF these images are diffraction-limited (FWHM=19 mas; Strehl=54\%; see Fig. 4). We do not use Lucky imaging in this study, since the long exposure (60s) images in Fig. 3 are much deeper (and almost as sharp) as those possible to obtain with Lucky in $60 \mathrm{~s}$ of telescope time.

\section{ASTROMETRY \& PHOTOMETRY}

All reduced (with SAA but not PRF corrected) 60s images of $\theta^{1}$ Ori $C_{1} C_{2}$ were analyzed with the DAOPHOT PSF fitting task allstar (Stetson 1987). The \pm 0.48 mas astrometric error of this very tight binary (where our $\sim 0.03$ mas platescale errors can be ignored) was estimated by the standard deviation of the astrometry differences between the three filters ([OI], r', $\mathrm{Ha}^{9}$ ) used. Our $\theta^{1}$ Ori $C_{1} C_{2}$ measurements of $32.64 \pm 0.48$ and $P A=206.31 \pm 0.17^{\circ}$ are compared to the interferometrically derived orbit in Fig. 5. We find reasonable agreement between the AO images and the interferometrically derived orbit of Kraus et al. (2009). For $\theta^{1}$ Ori $B_{1}, B_{2}, B_{3}, B_{4}$ and $\theta^{1}$ Ori $A_{1}, A_{2}$ the astrometry are summarized in Table 1 . The

\footnotetext{
${ }^{8}$ Section 5.6.1, ACS Instrument Handbook Cycle 19
} 
columns of Table 1 are self explanatory.

In the $\theta^{1}$ Ori $B$ group the PSF star used was the unsaturated $\theta^{1}$ Ori $B_{1}$ itself. Since all the members of the $\theta^{1}$ Ori $B$ group are located within $1^{\prime \prime}$ of $\theta^{1}$ Ori $B_{1}$ the PSF fit is particularly excellent there (there is no detectable change in PSF morphology due to anisoplanatic effects inside the $\theta^{1}$ Ori B group (Diolaiti et al. 2000)). Moreover, the residuals over the whole field were less than a few $\%$ after PSF subtraction. This is not really surprising given the quality of the nights combined with the fact that no star was further than $\sim 1^{\prime \prime}$ from the guide star. However, to minimize this affect, we only used the longer wavelength z' images reduced with SAA (taken on Dec 4, 2012) where anisoplanatic PSF effects were undetected. The relative positional accuracy is an excellent $\sim 0.2-1.4$ mas in radial separation. The $\sim 0.2-1.4$ mas separation errors are the resultant of the platescale uncertainty added in quadrature with the measurement uncertainty $(F W H M /(S / N))$. The errors are somewhat worse in the PA direction $(0.6-5$ mas $)$ due to a fixed \pm 0.3 degree conservative estimate of our absolute rotator uncertainly.

We can also compare our MagAO data to older (somewhat less accurate) images of the Trapezium B stars from Close et al. (2003c) who used AO images from Gemini and the 6.5m MMT and speckle images from the literature (Schertl et al. 2003). Even though these individual observations are of lower quality and Strehl than the MagAO ones (compare Figs. 6, 7, and 8 to that of MagAO in Fig. 9), the 15 years between these observations and those of MagAO can highlight even very small orbital motions of bound systems in the Trapezium. It also shows the very significant improvement in high Strehl AO now possible with Pyramid wavefront sensors and next generation adaptive secondary mirrors (ASMs).

A test to see how accurate our astrometry is over the last 15 years is to look at the scatter from a linear trend of the $\theta^{1}$ Ori $B$ group's motions. A comparison of our highly accurate positions with the historical positions from the literature is summarized in Table 1. Linear (weighted by astrometric error) fits to the data in Table 1 (Figures 10 to 15) yield the velocities shown in Table 1. The overall error in the relative proper motions is now an impressive $\lesssim 0.2$ mas $/$ yr in proper motion $(\lesssim 0.4 \mathrm{~km} / \mathrm{s})$ a factor of 2 improvement in accuracy when the VisAO positions are added into these calculations, compared to the last published values from Close et al. (2012b).

\footnotetext{
${ }^{9}$ While calibrating the throughput of the $H \alpha$ filter in the second commissioning run we found a faint companion to the famous transition disk young star HD 142527. The position of this companion at 83 mas and $130^{\circ} \mathrm{PA}$ was similar to a candidate companion found by aperture masking interferometry at the VLT by Biller et al. (2012) who measured $88 \pm 4$ mas, $133 \pm 3^{\circ}$. Hence, we report for the first time, that the existence of the close stellar companion HD 142527B is confirmed as real. Further details about this object are beyond the scope of this work but will be the focus of a future paper, Close et al. in prep.
} 


\section{ANALYSIS \& DISCUSSION}

With these accuracies it is now possible to determine whether these stars in the $\theta^{1}$ Ori $B$ group are bound together, or merely chance projections in this very crowded region. We adopt the masses of each star from the Siess Forestini \& Dougados (1997); Bernasconi \& Maeder (1996) tracks fit by Weigelt et al. (1999) where we find masses of: $B_{1} \sim 7 M_{\odot} ; B_{2} \sim 3 M_{\odot}$; $B_{3} \sim 2.5 M_{\odot} ; B_{4} \sim 0.2 M_{\odot} ; B_{5} \sim 7 M_{\odot} ; A_{1} \sim 20 M_{\odot} ; A_{2} \sim 4 M_{\odot} ;$ and $A_{3} \sim 2.6 M_{\odot}$. Based on these masses (which are similar to those adopted by Schertl et al. (2003)) we can comment on whether the observed motions are less than the escape velocities expected for simple face-on circular orbits.

Our combination of high spatial resolution and high signal to noise shows that there is very little significant motion in the $B_{1} B_{2}$ system over the last 15 years (as we might expect since the rotation of $B_{2}$ about the barycenter of the $B_{2} B_{3}$ system appears to be just canceling the motion of $B_{2}$ w.r.t. $B_{1}$ ). But of course it is really the barycenter of the tight $B_{2} B_{3}$ binary that would be in orbit around $B_{1}$. Hence the barycenter would have to show steady orbital motion if bound to $B_{1}$. Since $B_{2}$ is only $20 \%$ more massive than $B_{3}$ this means the $B_{2} B_{3}$ barycenter is currently 52 mas at PA $221.5^{\circ}$ from the center of $B_{2}$. In figures 10 and 11 we see that there is a small, yet significant, motion of the barycenter of $B_{2} B_{3}$ w.r.t. $B_{1}$ of some $0.80 \pm 0.18 \mathrm{mas} / \mathrm{yr}(1.6 \pm 0.3 \mathrm{~km} / \mathrm{s})$ and in PA by $0.030 \pm 0.044^{\circ} / \mathrm{yr}(1.0 \pm 1.0 \mathrm{~km} / \mathrm{s})$. Hence the motion of $B_{2} B_{3}$ is currently about $1.9 \pm 0.6 \mathrm{~km} / \mathrm{s}$ in the direction of PA $\sim 305^{\circ}$ (moving towards the WNW direction from $B_{1}$ ). This is the first time this motion has been detected. At this time it is not yet possible to prove this is true orbital motion, but given how close $B_{2} B_{3}$ is to $B_{1}$ this is likely orbital motion.

We have, of course, observed clear orbital motion (at $4.7 \pm 0.2 \mathrm{~km} / \mathrm{s}$ ) in the very tight $\theta^{1}$ Ori $B_{2} B_{3}$ system in almost pure PA (see Figure 13). In fact, now that we have observed over $20^{\circ}$ of PA rotation (with no significant change in separation), we have clear evidence of an "arc" of curvature of the system. The motion of the $B_{2} B_{3}$ binary is roughly consistent with a face-on, circular orbit (orbiting in the counterclockwise direction). A mildly elliptical orbit is also quite plausible given the very small amount of orbital phase observed to date.

Also we see linear orbital motion of $7.0 \pm 0.5 \mathrm{~km} / \mathrm{s}$ in the $\theta^{1}$ Ori $A_{1} A_{2}$ system (see Table 1). This is consistent with the motion seen by Grellmann et al. (2013). We know this is likely orbital motion since it is higher than the motion of unrelated stars in the cluster, due to their very close separation of just $0.19^{\prime \prime}$. 


\subsection{Is the $\theta^{1}$ Ori $B_{2} B_{3}$ System Physical?}

The relative velocity in the $\theta^{1}$ Ori $B_{2} B_{3}$ system (in the plane of the sky) is now more accurate by $\sim 10 \times$ compared to that of Close et al. (2003c) and by $\sim 2 \times$ compared to Close et al. (2012b). Our new velocity of $4.7 \pm 0.2 \mathrm{~km} / \mathrm{s}$ is consistent, but with much lower errors, with the $\sim 4.2 \pm 2.1 \mathrm{~km} / \mathrm{s}$ of Close et al. (2003c) (this velocity is in the azimuthal direction; see Figure 13). This is a reasonable $V_{\text {tan }}$ since an orbital velocity of $\sim 6.7 \mathrm{~km} / \mathrm{s}$ is expected from a face-on circular orbit from a $\sim 5.5 M_{\odot}$ binary system like $\theta^{1}$ Ori $B_{2} B_{3}$ with a $49 \mathrm{AU}$ projected separation (implying an orbital period of order $\sim 200 \mathrm{yr}$ ). This theoretical value of $\sim 200 \mathrm{yr}$ is close to the $302 \pm 16 \mathrm{yr}$ orbit that comes from assuming the current measured angular (PA) velocity stays constant. It is worth noting that this velocity is also greater than the $\sim 3 \mathrm{~km} / \mathrm{s}$ Hillenbrand \& Hartmann (1998) velocity dispersion of the cluster.

Our observed velocity of $1.19 \pm 0.06^{\circ} / \mathrm{yr}$ is $\sim 10 \times$ more accurate than that of Close et al. (2003c). This primarily azimuthal motion strongly suggests a curving orbital arc of $B_{3}$ orbiting $B_{2}$ counterclockwise.

\subsection{Is the $\theta^{1}$ Ori B Group Stable Long Term?}

The barycenter of $B_{2} B_{3}$ is moving at a low $1.9 \pm 0.6 \mathrm{~km} / \mathrm{s}$ in the plane of the sky w.r.t. to $B_{1}$ (itself a very tight pair with $B_{5}$ ) where the escape velocity $V_{\mathrm{esc}} \sim 6 \mathrm{~km} / \mathrm{s}$ for this massive system $\left(\sim 20 M_{\odot}\right)$. Hence these two pairs are likely gravitationally bound together. This is the first effort that has measured this small barycenter velocity definitively. Hence, we can say that these two pairs currently form a rare bound "mini-cluster" of young massive stars.

\subsubsection{Is the Orbit of $\theta^{1}$ Ori $B_{4}$ Stable?}

The two AO measurements of Close et al. (2003c) (and the one speckle detection of Schertl et al. (2003)) did not detect a significant velocity of $B_{4}$ w.r.t. $B_{1}: 2 \pm 11 \mathrm{~km} / \mathrm{s}$. However, our much better data and timeline between the LBT epoch and the excellent MagAO observations has shed some light on the question of B4 orbiting B1. As is clear from Figures 14 \& 15, there appears to be a real velocity of $4.1 \pm 1.2 \mathrm{~km} / \mathrm{s}$ detected. This is greater than the random velocity of the cluster yet below the escape velocity of $\sim 6 \mathrm{~km} / \mathrm{s}$, this points towards $B_{4}$ being also gravitationally bound member of the $\theta^{1}$ Ori $\mathrm{B}$ group. Again we are observing almost pure motion in PA (0.181 $/$ yr counterclockwise). Assuming 
a simple face-on orbit we would expect a very rough period of $\sim 2000 \pm 700$ years for $B_{4}$ to orbit $B_{1}$ given an average angular velocity of $0.181^{\circ} / \mathrm{yr}$.

\subsection{A Possible Model for the Orbits of the $\theta^{1}$ Ori $B$ Group}

It is tempting to define a circular orbit baseline model of the $\theta^{1}$ Ori $B$ system with the center as the very tightly bound 6.47 day massive $\left(\sim 14 M_{\text {sun }}\right) 0.13$ AU spectroscopic binary $B_{1} B_{5}$ which we cannot spatially resolve. Around this center is the low mass $\sim 0.2 M_{\text {sun }} B_{4}$ some $\sim 254$ AU away which orbits every $\sim 2000 \pm 700$ years in a roughly face-on circular orbit. Then, further out, the tight 49 AU binary $B_{2} B_{3}$ rotates every $302 \pm 16$ years around its barycenter with roughly a face-on circular orbit. However, the co-planar geometry is broken by the orbit of this barycenter around $B_{1}$. It appears that the $B_{2} B_{3}$ barycenter is moving in a bound orbit to WNW $\left(\mathrm{PA} \sim 305^{\circ}\right)$. This motion cannot be in a simple face-on circular orbit, and so must be (if close to circular) inclined by about $\sim 30^{\circ}$, but many other elliptical orbits are also possible. We simply do not have enough of time baseline to understand the fine details of this orbit today. If we simply assume it is a inclined circular orbit then it has roughly a $\sim 820 A U$ (deprojected) separation from $B_{1}$ and a period of some $\sim 11,000$ years.

See Figs 16 and 17 for illustrations of what these obits would look like if they are all close to circular. It is interesting to note, that once the true (deprojected) separation of $B_{2} B_{3}$ is considered, the group seems more hierarchical than reported in Close et al. (2012b). For example, the ratio of the 3 main periods are $P_{23}: P_{1 / 4}: P_{1 / 23}=1: 7: 36$ so that there is almost an order of magnitude separating each period. This large spread of orbital periods will lend some stability to this "mini-cluster". On the other hand, $B_{4}$ 's very low mass, its intermediate period, and its location w.r.t. to the other four groups members makes it highly unlikely that $B_{4}$ is on a long-term stable orbit within the group. It is very likely that an interaction between the much more massive $B_{2} B_{3}$ and $B_{4}$ will eject $B_{4}$ in the future -leading to a slightly more tightly bound "mini-cluster" without $B_{4}$. As we will discuss in the next section, even the much more massive $B_{3}$ may not even be stable in the long-term.

\subsubsection{Is the orbit of $B_{3}$ around $B_{2}$ and of $B_{5}$ around $B_{1}$ stable in the long-term?}

Close et al. (2012b) noted that the distance $D_{B_{1} B_{5}} \sim 3 \times 10^{-4} \times D_{B_{1} B_{5} B_{2} B_{3}}$ and thus the very tight $(0.13 \mathrm{AU})$ tight $B_{1} B_{5}$ system is, of course, very stable. More interesting is the case of $B_{2} B_{3}$. Their de-projected distance is not very small compared to their projected distance (D) from the $B_{1} B_{5}$ pair: $D_{B_{2} B_{3}} \sim 0.06 \times D_{B_{1} B_{5} B_{2} B_{3}}$. Thus the stability of the $B_{2} B_{3}$ 
orbit needs a more detailed analysis since it is possible that $B_{3}$ may be ejected in the future.

The orbital period of the two binaries w.r.t. each other is $P_{1 / 23} \sim 11000 \mathrm{yrs}$, while the orbital period of $B_{3}$ w.r.t $B_{2}$ amounts to $P_{2 / 3} \sim 300 \mathrm{yrs}$. For the calculation of both periods, we have assumed the masses as given above, and circular orbits in the plane of the sky (except for $B_{1} B_{3}$ which is inclined at $\sim 30^{\circ}$ ). This leads to a period ratio $X=$ $P_{1 / 23} / P_{2 / 3} \sim 36$. Eggelton \& Kiseleva's stability criterion requires $X \geq X_{\text {crit }}=10.08$ for the masses in the B group. This means that within the accuracy limits of our investigation,

the binary $B_{2} B_{3}$ is likely stable (different from the marginal stability found in Close et al. $(2012 \mathrm{~b}))$. The stability criterion depends also on the orbits' eccentricities. However, mild eccentricities of the order of $e \sim 0.1$ (as can be expected to develop in hierarchical triple systems; see, e.g., Georgakarakos 2002), can make the B group unstable. However, the $\theta^{1}$ Ori B system seems to be a good example of a highly dynamic star formation "minicluster" which might, in the future, eject the lowest-mass member(s) through dynamical decay (Durisen. Sterzik, \& Pickett 2001).

\section{Conclusions}

In this study we utilized the new high-order (585 actuator) Magellan Adaptive Optics system (MagAO) to obtain very high-resolution science in the visible with MagAO's VisAO CCD camera. In the median seeing conditions of Magellan $\left(0.5-0.7^{\prime \prime}\right)$ we found that MagAO delivers individual short exposure images as good as 19 mas optical resolution. Due to residual $60 \mathrm{~Hz}$ vibrations, long exposure $(60 \mathrm{~s}) \mathrm{r}$ ' $(0.63 \mu \mathrm{m})$ images are slightly coarser at FWHM $=23-29$ mas (Strehl $\sim 28 \%)$ with bright $(R<9$ mag) guide stars. These are the highest resolution filled-aperture images published to date. Images of the young $(\sim 1 \mathrm{Myr})$ Orion Trapezium $\theta^{1}$ Ori A, B, and C cluster members were obtained with the VisAO camera. In particular, the 32 mas binary $\theta^{1}$ Ori $C_{1} C_{2}$ was easily resolved in non-interferometric images for the first time. Relative positions of the bright trapezium binary stars were measured with 0.6-5.0 mas accuracy. We now are sensitive to relative proper motions of just $\sim 0.2$ mas/yr $(\sim 0.4 \mathrm{~km} / \mathrm{s}$ at $414 \mathrm{pc})-$ this is a $\sim 2-10 \times$ improvement in velocity accuracy compared to previous efforts. We now detect clear orbital motions of $\theta^{1}$ Ori $B_{2} B_{3}$ and $A_{1} A_{2}$ of $4.7 \pm 0.2$ $\mathrm{km} / \mathrm{s}$ and $7.1 \pm 0.5 \mathrm{~km} / \mathrm{s}$, respectively. For the first time, we see clear motion of the barycenter of $\theta^{1}$ Ori $B_{2} B_{3}$ in about $\theta^{1}$ Ori $B_{1}$. All five members of the $\theta^{1}$ Ori $B$ system appear likely a gravitationally bound "mini-cluster", but we find that not all the orbits can be both circular and co-planar. The very lowest mass member of the $\theta^{1}$ Ori $B$ system $\left(B_{4} ;\right.$ mass $\left.\sim 0.2 M_{\odot}\right)$ has a very clearly detected motion (at $4.1 \pm 1.3 \mathrm{~km} / \mathrm{s}$; correlation=99.9\%) w.r.t $B_{1}$. Previous work has suggested that $B_{4}$ and $B_{3}$ are both on long-term unstable orbits and will be ejected 
from this "mini-cluster". However, our new "baseline" model of the $\theta^{1}$ Ori $B$ system suggests a more hierarchical system than previously thought, and so the ejection of $B_{4}$ may not occur for many orbits, and $B_{3}$ may be stable against ejection long-term. This "ejection" process of the lowest mass member of a "mini-cluster" could play a major role in the formation of low mass stars and brown dwarfs.

\section{FUTURE OBSERVATIONS}

Future observations are required to see, if indeed, these stars continue to follow orbital arcs around each other proving that they are interacting with one another. In addition, future observations of the $\theta^{1}$ Ori $B_{4}$ positions would help deduce if it is on a marginally stable orbit given its somewhat "non-hierarchical" location in the B group.

Future observations should also try to determine the radial velocities of these stars. Once radial velocities are known one can calculate the full space velocities of these stars. Such observations will require both very high spatial and spectral resolutions. This might be possible with such instruments like the AO fed ARIES echelle instrument at the MMT.

We thank the whole Magellan Staff for making this wonderful telescope possible for use with our AO system. We would especially like to thank Povilas Palunas (for help over the entire MagAO commissioning run). Juan Gallardo (and his professional team), Patricio Jones, Emilio Cerda, Felipe Sanchez, Gabriel Martin, Maurico Navarrete, Jorge Bravo and the whole team of technical experts helped do many exacting tasks in a very professional manner. Glenn Eychaner, David Osip and Frank Perez all gave expert support which was fantastic. Also thanks to Victor, Maurico, and Hugo for running the telescope so well. It is a privilege to be able to commission an $\mathrm{AO}$ system on such a fine telescope and site. Thanks from the whole MagAO team. And thanks to Miguel Roth, Francisco Figueroa, Roberto Bermudez, Sergio Veliz and, of course, Mark Philips for making this all happen - and very smoothly - despite the large AO team that was needed at the mountain and all the headaches and extra work it created for the LCO staff. We also thank the teams at Steward Observatory Mirror Lab/CAAO (University of Arizona), Microgate (Italy) and ADS (Italy) for building such a great ASM. The MagAO ASM was developed with support from the NSF MRI program. The MagAO PWFS was developed with help from the NSF TSIP program and the Magellan partners. The Active Optics guider was developed by Carnegie Observatories with custom optics from the MagAO team. The VisAO camera and commissioning was supported with help from the NSF ATI program. LMC's research was supported by NSF AAG and NASA Origins of Solar Systems grants. JRM is grateful for 
the generous support of the Phoenix ARCS Foundation. KM was supported under contract

with the California Institute of Technology (Caltech) funded by NASA through the Sagan Fellowship Program.

\section{REFERENCES}

Abt H.A., Wang R., Cardona O., 1991, ApJ, 367, 155

Baranec, C.; Riddle, R.; Ramaprakash, A. N.; Law, N.; Tendulkar, S.; Kulkarni, S.; Dekany, R.; Bui, K.; Davis, J.; Burse, M.; Das, H.; Hildebrandt, S.; Punnadi, S.; Smith, R. Proc SPIE Volume 8447, id. 844704-844704-11

Bate, M.R., Bonnell, I.A., Bromm, V. 2002, MNRAS, 332, L65

Bate, M.R., Bonnell, I.A., Bromm, V. 2003, MNRAS, 277, 362

Bate, M.R. 2009, MNRAS, 392, 590

Bate, M.R. 2011, MNRAS, in press

Bazzon, Andreas; Gisler, Daniel; Roelfsema, Ronald; Schmid, Hans M.; Pragt, Johan; Elswijk, Eddy; de Haan, Menno; Downing, Mark; Salasnich, Bernardo; Pavlov, Alexey; Beuzit, Jean-Luc; Dohlen, Kjetil; Mouillet, David; Wildi, Franois (2012) Proceedings of the SPIE, Volume 8446, id. 844693-844693-14

Bernasconi P.A., \& Maeder A. 1996, A\&A, 307, 829

Biller, B., et al. 2012, ApJ, 753, L38

Brusa, G., et al. 2003a, Proc. SPIE 4839, 691.

Brusa, G., et al. 2003b, Proc. SPIE 5169, 26

Burgasser, A. et al. 2003, ApJ, 586, 512

Burgasser, A., Reid, L.N., Siegler, N., Close, L., Allen, P., Lowerance, P., Gizis, J. (2007),Protostars and Planets V, B. Reipurth, D. Jewitt, and K. Keil (eds.), University of Arizona Press, Tucson, 951 pp., p.427-441

Burrows, A., Hubbard, W. B., Lunine, J. I., Marley, M. S., Saumon, D. 2000, Protostars and Planets IV (Tucson: University of Arizona Press, eds Mannings, V., Boss, A.P., Russell, S. S.), p. 1339 
Bouy, H., Brandner W., Martín, E., Delfosse, X., Allard, F., \& Basri, G. 2003, AJ, 126, 1526

Bossi M., Gaspani A., Scardia M., Tadini M., 1989, A\&A, 222, 117

Chabrier, G., Baraffe, I., Allard, F., \& Hauschildt, P. 2000, ApJ, 542, 464

Close, L.M., Roddier, F.J., Roddier, C.A., Graves, J.E., Northcott, M.J., Potter, D. 1998, Proc. SPIE Vol. 3353, p. 406-416. Adaptive Optical System Technologies, D. Bonaccini, R.K. Tyson, Eds

Close, L. M. 2000, Proc. SPIE Vol. 4007, p758-772. Adaptive Optical Systems Technology, P.L. Wizinowich, Ed.

Close, L.M. et. al. 2002a, ApJ, 566, 1095.

Close, L.M. et. al. 2002b, ApJ, 567, L53.

Close, L.M., Siegler, N., Freed, M., Biller, B. 2003a ApJ, 587, 407

Close, L.M. et al. 2003b ApJ, 598, 35.

Close, L.M. et al. 2003c ApJ, 599, 537.

Close, L.M. et al. 2007 ApJ, 665, 736.

Close, L.M. et al. 2012a Proc. SPIE, Volume 8447, id. 84470X-84470X-16

Close, L.M. et al. 2012b ApJ, 749, article id. 180.

Dekany, R. et al. Third International Conference on Adaptive Optics for Extremely Large Telescopes, in prep.

Diolaiti, E., Bendinelli, O. Bonaccini, D.; Close, L Currie, D. Parmeggiani, G. 2000, A\&AS 147,335

Durisen, R.H., Sterzik, M.F., \& Pickett, B.K. 2001, A\&A, 371, 952

Duquennoy, A., Mayor, M. 1991, A\&A, 248, 485

Eggelton P., Kiseleva L., 1995, ApJ, 455, 640

Esposito, S. et al. proc. 2011, SPIE 8149, 814902-10

Esposito, S. et al. proc. 2010a SPIE 7736, 773609-12

Esposito, S. et al. proc. 2010b Applied Optics, 49, issue 31, p. G174 
Esposito, S. et al. 2012 proc. SPIE Volume 8447, article id. 84470U

Fischer, D. A., Marcy, G. W. 1992, ApJ, 396, 178

Freed, M., Close, L.M., \& Siegler, N. 2003, ApJ, 584, 453

Garrel, Vincent; Guyon, Olivier; Baudoz, Pierre, PASP, 124, 861.

Genzel R., Stutzki J., 1989, ARA\&A 27, 41

Graves, J.E., Northcott, M.J., Roddier, F.J., Roddier, C.A., Close, L.M. 1988, Proc. SPIE Vol. 3353, p. 34-43. Adaptive Optical System Technologies, D. Bonaccini, R.K. Tyson, Eds.

Grellmann, R., Preibisch, T., Ratzka, T., Kraus, S. Helminiak, K.G., Zinnecker, H. 2013, A\&A 550, 82.

Gizis, J.E. et al. 2003, ApJ, 120, 1085

Hillenbrand L.A., \& Carpenter J. 2000 ApJ, 540, 236

Hillenbrand L.A., \& Hartmann L.W. 1998 ApJ, 492, 540

Hinz J.L., McCarthy D.W., Simons, D.A., Henry T.J., Kirkpatrick J.D., McGuire P.C. 2002, AJ, 123, 2027

Hinz P.M. et al. 2010, ApJ716 417

Hodapp, K.-W., Hora, J. L., Hall, D. N. B., Cowie, L. L., Metzger, M., Irwin, E., Vural, K., Kozlowski, L. J., Cabelli, S. A., Chen, C. Y., Cooper, D. E., Bostrup, G. L., Bailey, R. B., Kleinhans, W. E. 1996, New Astronomy, 1, 177

Kiseleva L.G., Eggelton P.P., Anosova J.P., 1994, MNRAS 267, 161

Kiseleva L.G., Eggelton P.P., Orlov V.V., 1994, MNRAS 270, 936

Kraus, S. et al. 2007, A\&A 466, 649

Kraus, S. et al. 2009, A\&A 497, 195

Kohler, R. et al. 2006, A\&A 458, 461

Kopon, D. et al. 2012, SPIE, Volume 8447, id. 84473D-84473D-15.

Law, N. M.; Mackay, C. D.; Dekany, R. G.; Ireland, M.; Lloyd, J. P.; Moore, A. M.; Robertson, J. G.; Tuthill, P.; Woodruff, H. C. 2009, ApJ 692, 924. 
Lloyd-Hart M. 2000, PASP 112, 264

Males, J.R. et al. 2012, SPIE, Volume 8447, id. 844742-844742-12.

Males, J.R. et al. 2013 in prep.

McCaughrean M.J, \& Stauffer J.R., 1994, AJ, 108, 1382

McCaughrean M.J 2000, The Formation of Binary Stars, Proceedings of IAU Symp. 200, held 10-15 April 2000, in Potsdam, Germany, Edited by Hans Zinnecker and Robert D. Mathieu, 2001, p. 169.

McCarthy C., Zuckerman, B. (2004), AJ 127, 2871

McCarthy D.W. et al. 1998, Proc. SPIE 3354750

McDonald, J. M., \& Clarke, C. J. 1993, MNRAS, 262, 800

Menten K. M.; Reid, M. J.; Forbrich, J.; Brunthaler, A. 2007, A\&A 474, 515.

Morzinski, Katie; Johnson, Luke C.; Gavel, Donald T.; Grigsby, Bryant; Dillon, Daren; Reinig, Marc; Macintosh, Bruce A. 2010 Proceedings of the SPIE, Volume 7736, pp. 77361O-77361O-16

Morzinski, Katie; et al. 2013 in prep.

Parker, R.J., Goodwin, S., Allison, R.J. 2011, MNRAS418, 2565

Petr M.G., Du Foresto V., Beckwith S.V.W., Richichi A., McCaughrean M.J. 1998, ApJ, 500,825

Potter, D. et al. 2002a ApJ, 567, 113

Reid, I. N., Gizis, J.E., Kirkpatrick, J.D., Koerner, D. W. 2001a, AJ, 121, 489

Reid, I. N., Burgasser, A. J., Cruz, K. L., Kirkpatrick, J. D., Gizis, J. E. 2001b, AJ, 121, 1710

Reipurth, B. \& Clarke, C. 2001, AJ, 122, 432

Ricci, Luca; Robberto, M.; Soderblom, D. R.; Kozhurina-Platais, V. 2007, BAAS 211, 8923

Schertl, D., Balega, Y.Y., Preibisch, Th., \& Weigelt, G. 2003, A\&A, 402, 267

Siegler, N., Close, L.M., Mamajek, E., Freed, M. 2003, ApJ, 598, 1265 
Siess L., Forestini M., Dougados C., 1997, A\&A 324, 556

Simon, M., Close, L.M., \& Beck, T. 1999, AJ, 117, 1375

Sterzik, M. F., \& Durisen, R. H. 1998 A\&A, 339, 95

Stetson, P. B. 1987, PASP, 99, 191

Thomas-Osip Joanna E.; McCarthy, Patrick; Prieto, Gabriel; Phillips, Mark M.; Johns, Matt 2010,Proceedings of the SPIE, Volume 7733, pp. 77331L-77331L-10.

Wainscoat R. J., \& Cowie, L.L. 1992, AJ, 103, 332.

Weigelt G., Balega, Y., Preibisch T., Schertl D., Scholler M., Zinnecker H. 1999, A\&A, 347, L15

Wildi F., Brusa G., Riccardi A., Lloyd-Hart M., Martin H.M., L.M. Close 2003, proc. SPIE 4839,155

Wildi F. Brusa G., Lloyd-Hart M., Martin H.M., L.M. Close, Riccardi A. 2003b, proc SPIE $5169,17$. 
Table 1. High Resolution Observations of the $\theta^{1}$ Ori B \& A groups

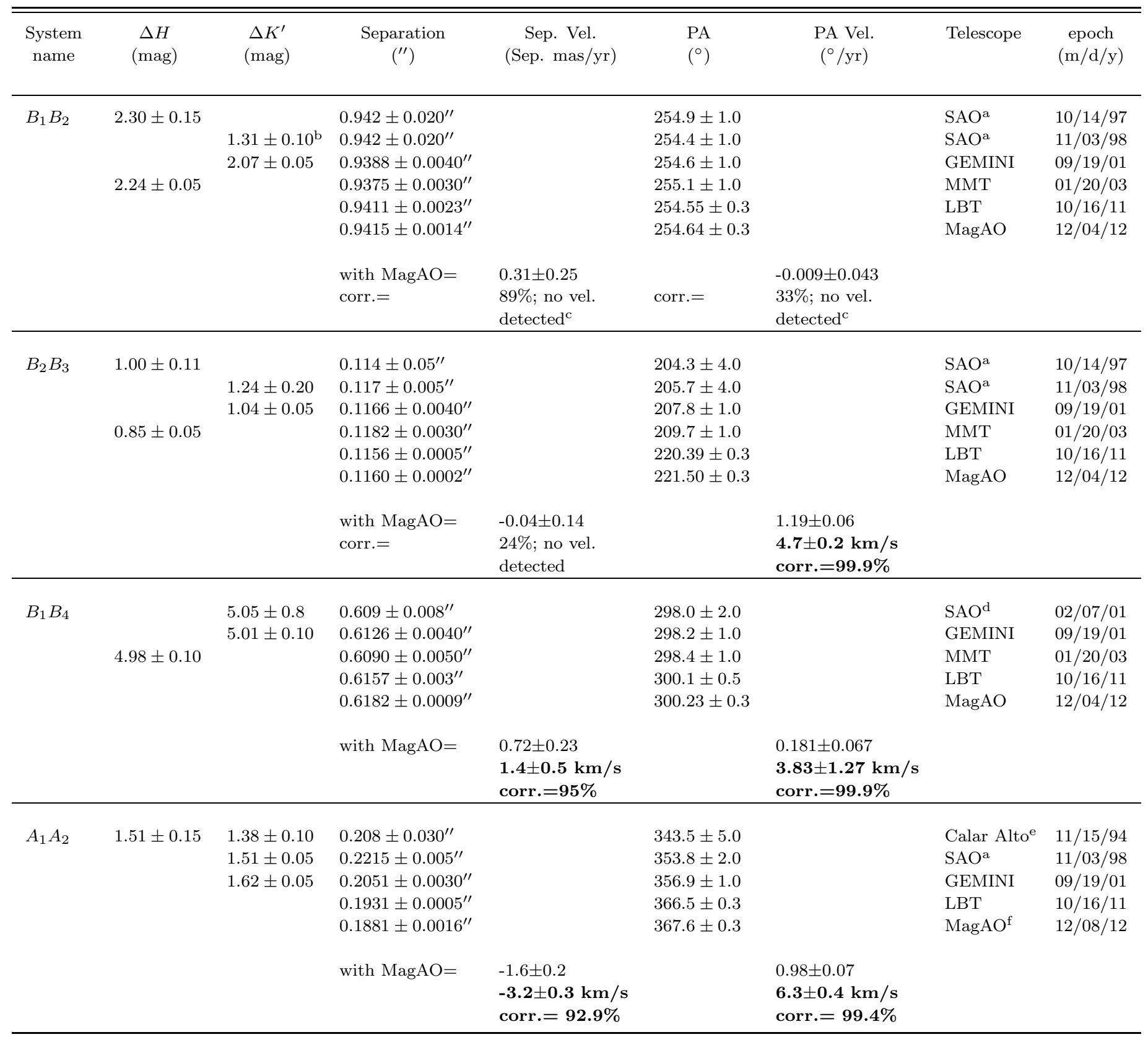

${ }^{a}$ speckle observations of Weigelt et al. (1999).

b these low $\Delta K$ values are possibly due to $\theta^{1}$ Ori $B_{1}$ being in eclipse during the 11/03/98 observations of Weigelt et al. (1999).

${ }^{\mathrm{c}}$ Note there is velocity detected from $B_{1}$ w.r.t. the barycenter of the $B_{2} B_{3}$ binary see fig 10 and fig 11

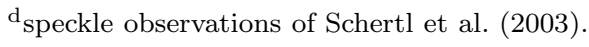

${ }^{\text {e }}$ speckle observations of Petr et al. (1998).

${ }^{\mathrm{f}} A_{1} A_{2}$ Data from Ks image from the MagAO/Clio2 NIR camera (Morzinski et al. 2013 in prep). 


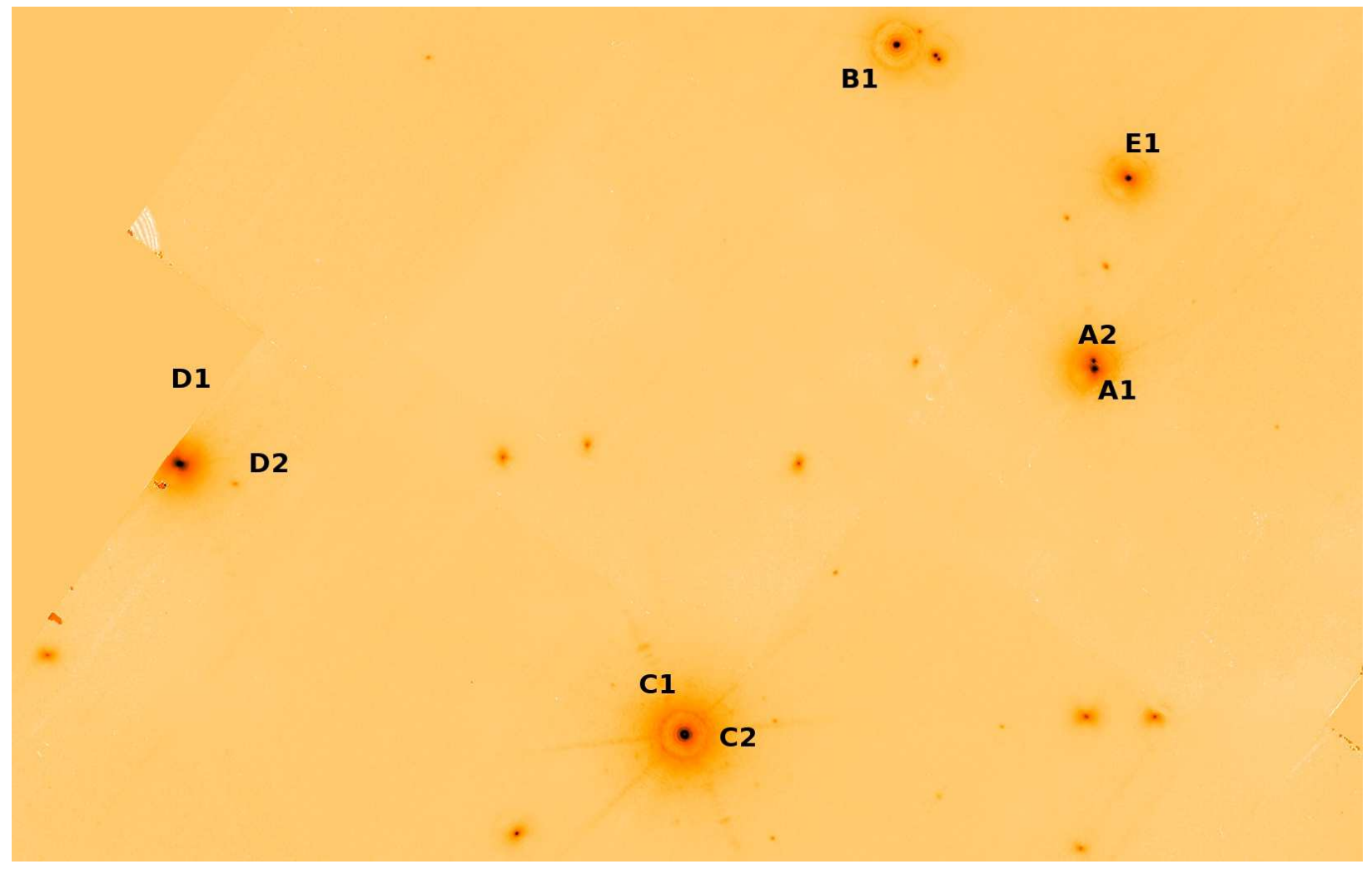

Fig. 1.- The locations and nomenclature of Close et al. (2003c) of the $\theta^{1}$ Ori Trapezium stars as imaged over $\sim 35 \times 30^{\prime \prime}$ FOV at the LBT with LBTAO/PISCES in [FeII] (reproduced from Close et al. $(2012 \mathrm{~b})$ )). Logarithmic color scale. North is up and east is left. Note that the object " $A_{1}$ " is really a spectroscopic binary $\left(A_{1} A_{3}\right)$; where the unseen companion $A_{3}$ is separated from $A_{1}$ by $1 \mathrm{AU}$ (Bossi et al. 1989). The B group is shown in more detail in Figs. 6] - 9. It is not currently clear if $D_{2}$ is physically related to $D_{1}$. $E_{1}$ appears to be a single star. No new faint companions were discovered (at $>5 \sigma$ ) around any of the Trapezium stars in this study. 


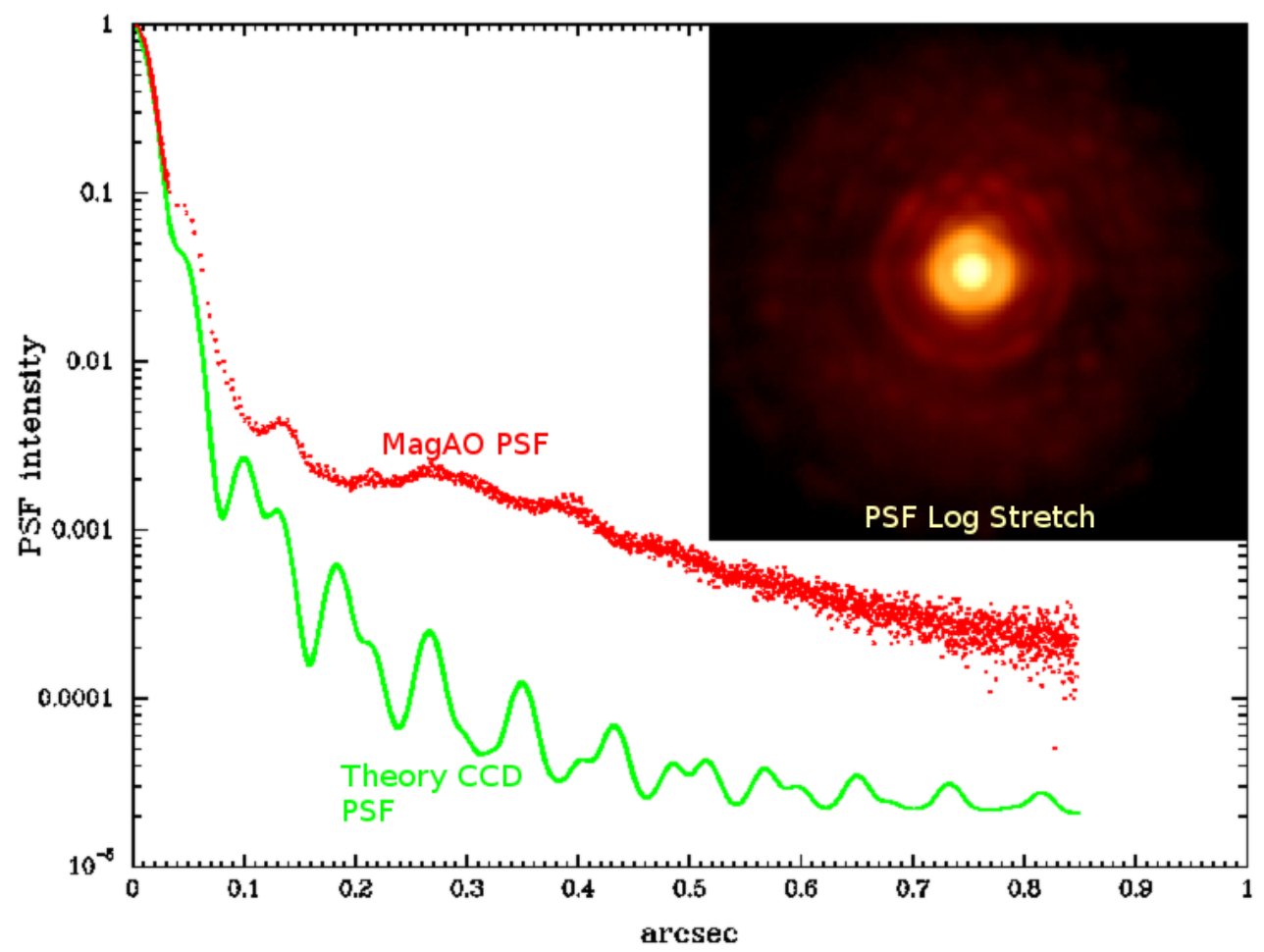

Fig. 2.- The radial profile (red points) of a deep (300s) MagAO $Y_{\text {short }}(0.98 \mu m)$ PSF on a bright ( $\mathrm{V}=5 \mathrm{mag}$ ) star closed-loop at $990 \mathrm{~Hz}$ with 250 modes in $0.6^{\prime \prime} \mathrm{V}$ band seeing (from Males et al. (2013)). Insert: a log10 Stretch of the PSF. There was no post-detection processing of any of the data (no SAA, no Lucky imaging or frame selection applied). The theoretical MagAO PSF profile as imaged by the E2V CCD47 (Strehl 100\%). A detailed comparison of the observed PSF to theory with our CCD47 (including dark current and PRF) shows that we reached a Strehl of $43 \%$ or $140 \mathrm{~nm}$ rms optical wavefront error. 


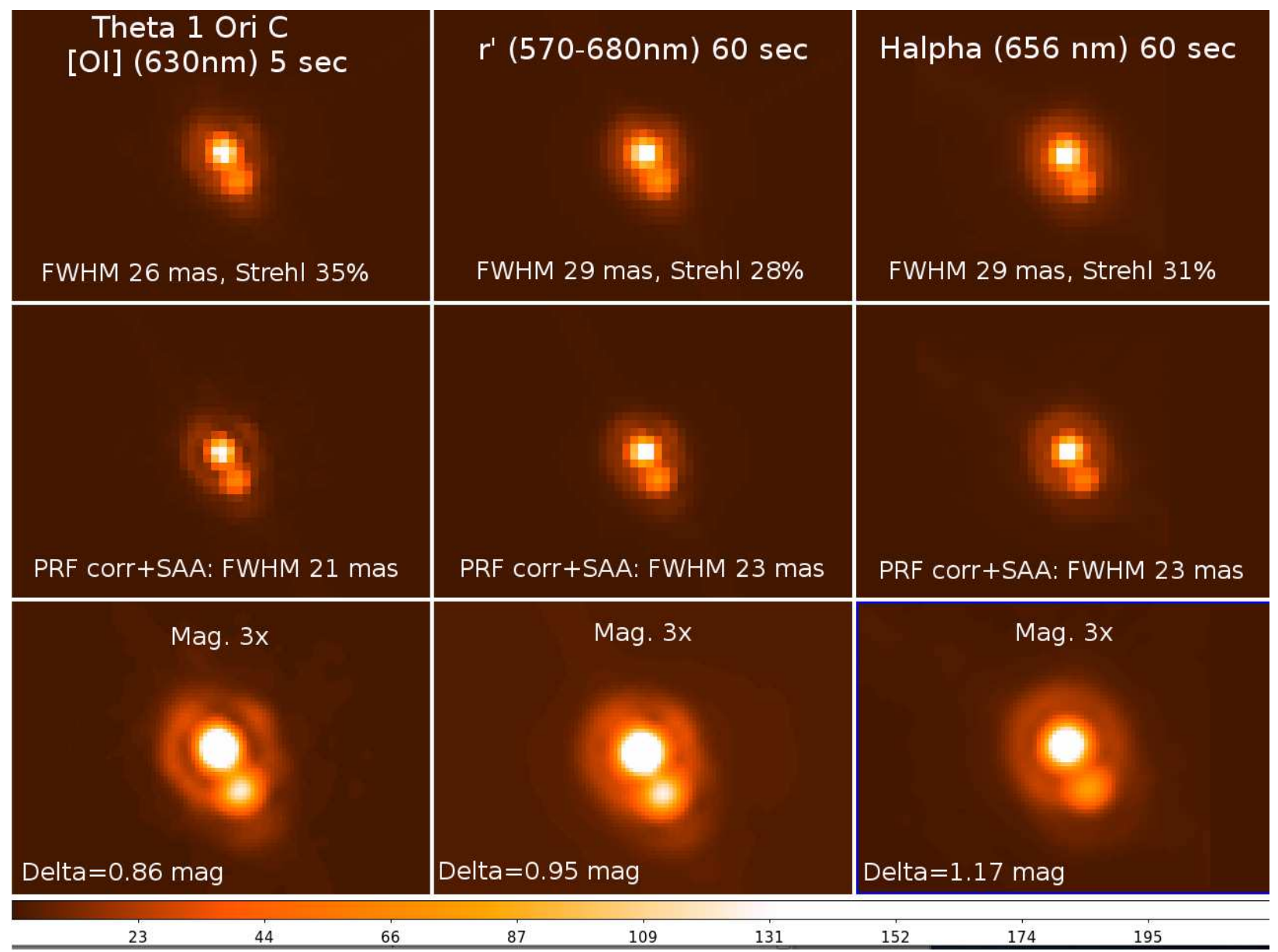

Fig. 3. - Top row: the central ionizing binary of the Trapezium: $\theta^{1}$ Ori $\mathrm{C}$ as imaged with MagAO's VisAO CCD camera in different filters. Note the excellent resolution in the raw 60 second image. We note that no post-detection shift and add (SAA) was applied, nor was there any frame selection used to produce these top row images. Typically we achieved resolutions of $0.026-0.029^{\prime \prime}$ and Strehls of $28-35 \%$ in $0.5-0.7^{\prime \prime} \mathrm{V}$-band seeing. Middle row: the same data as the top row, except the images have been post-detection aligned (SAA) and the pixel response function (PRF) has been removed. This improved image resolution by $\sim 5-6$ mas. Bottom row: the row above is magnified by $3 \times$ to better disply the data of the middle row. These are the highest resolution, deep, images ever obtained to our knowledge. 

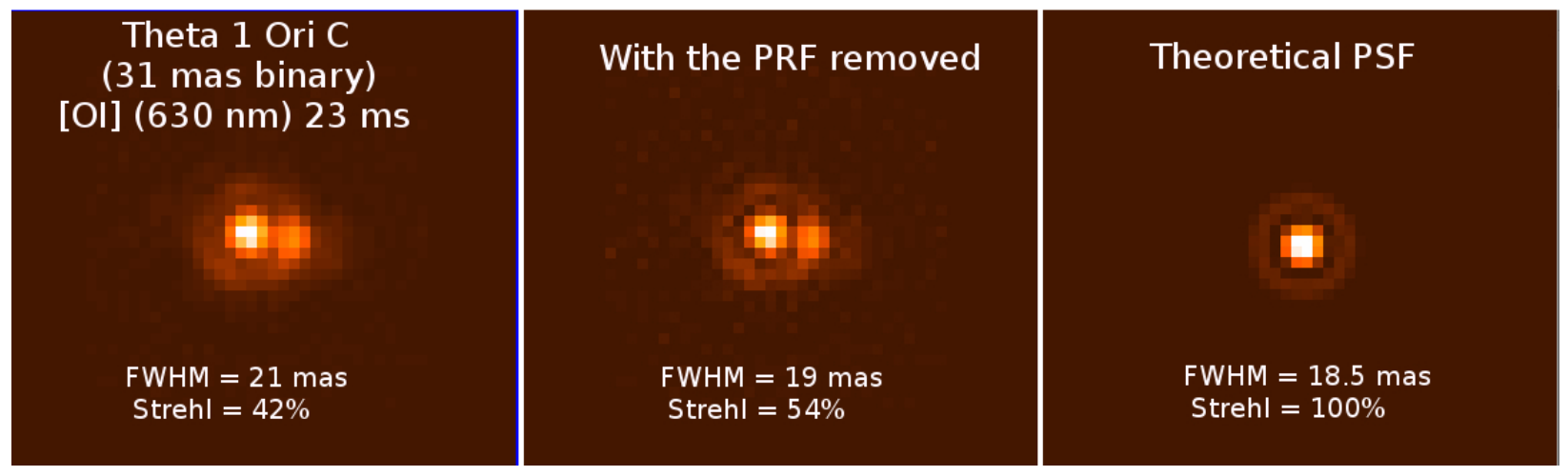

Fig. 4.- An excellent short exposure single image of $\theta^{1}$ Ori $\mathrm{C}$ at [OI] $(630 \mathrm{~nm})$. On the left is the raw image with a resolution of $0.021^{\prime \prime}$, Strehl $42 \%$. Then the VisAO CCD's PRF is removed in the middle box and so the resolution is restored to the true value entering the CCD of $0.019^{\prime \prime}$. These are the highest resolution short exposure images ever obtained on any telescope to our knowledge. 


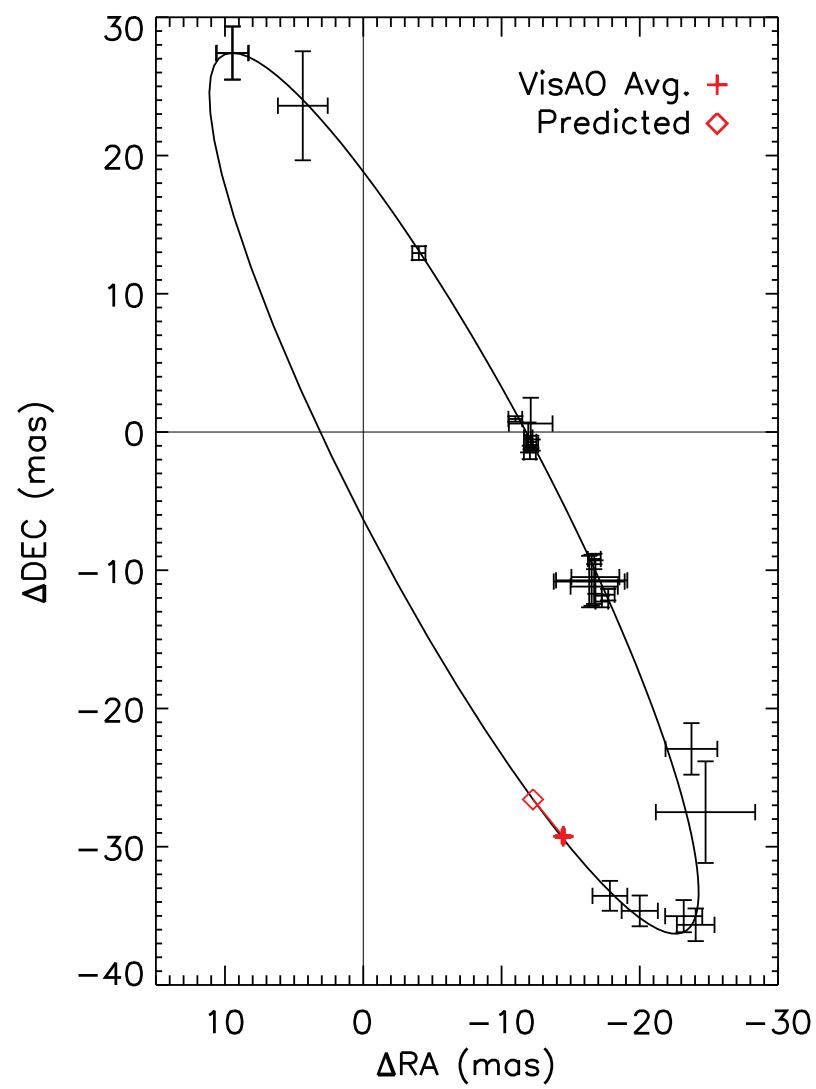

Fig. 5.- PSF fitting photometry of the [OI], r', and $H_{\alpha}$ images in Fig. 3 of $\theta^{1}$ Ori $C_{1} C_{2}$ with a $44 \%$ Strehl theoretical PSF gave a good fit to both binary components in all cases. For Dec 12, 2012 UT we find separation is $32.64 \pm 0.48$ mas and PA is $206.31 \pm 0.17^{\circ}$. Here we plot this position (in red) against the interferometric orbit of Kraus et al. (2009). The agreement with the predicted position of $C_{2}$ w.r.t. $C_{1}$ is reasonable given the uncertainty of the orbital solution. However, more VisAO astrometry at this level of accuracy following this poorly sampled side $\left(20^{\circ}<\mathrm{PA}<210^{\circ}\right)$ of the orbit over the next few years would certaintly lead to a better orbital solution than is known today. 


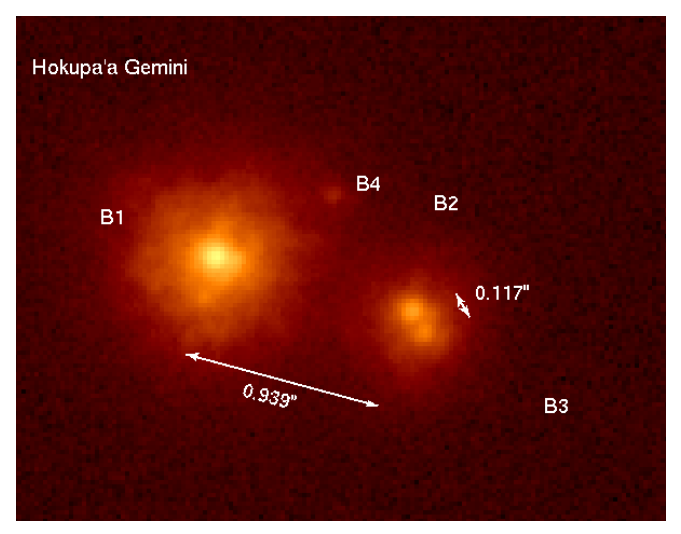

Fig. 6. - The $8 \mathrm{~m}$ Gemini/Hokupa'a images of the $\theta^{1}$ Ori B group in the $K^{\prime}$ band (09/19/01; from Close et al. (2003c)). Resolution 0.085". Log scale. North is up and East is left.

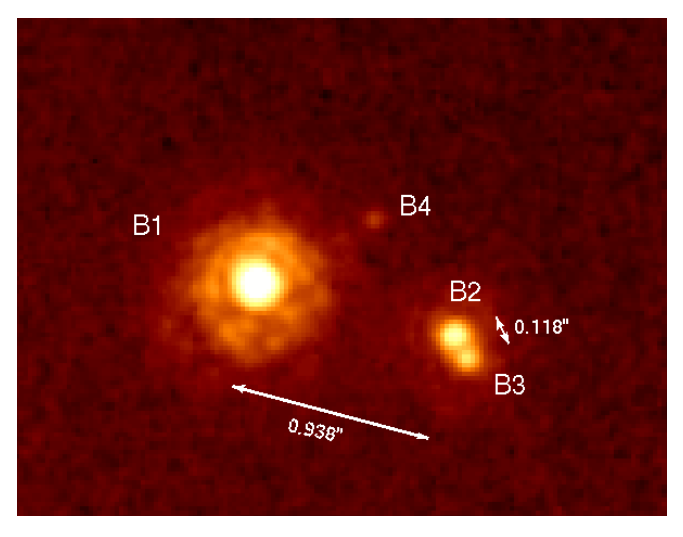

Fig. 7.- Detail of the $\theta^{1}$ Ori B group as imaged at $0.077^{\prime \prime}$ (Strehl $>20 \%$ ) resolution (in the $\mathrm{H}$ band) with the MMT AO system (01/20/03) from Close et al. (2003c). 


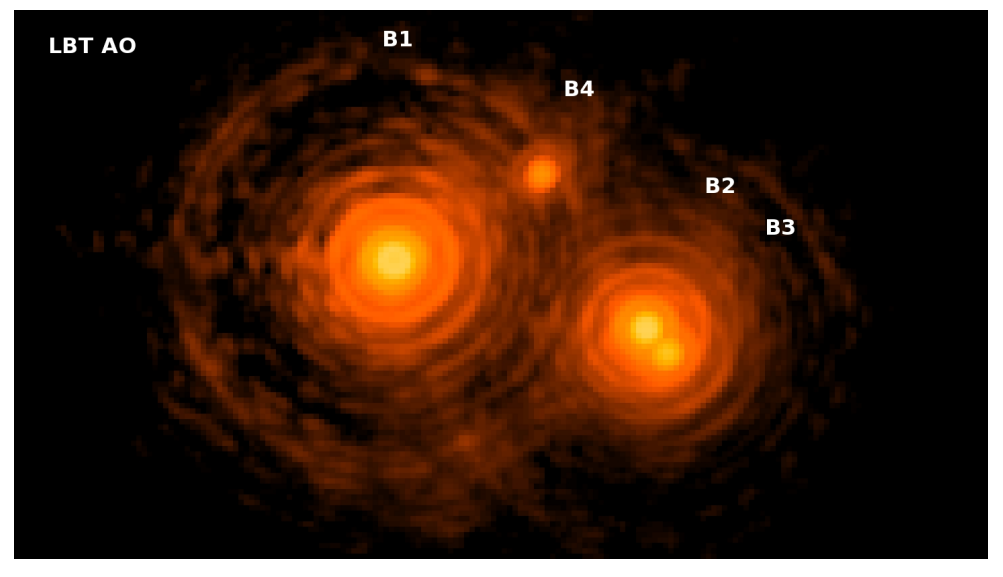

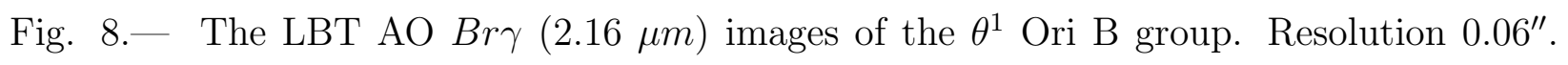
Logarithmic color scale. North is up and east is left. Strehl is $\sim 75 \%$ (from Close et al. $(2012 \mathrm{~b}))$. 


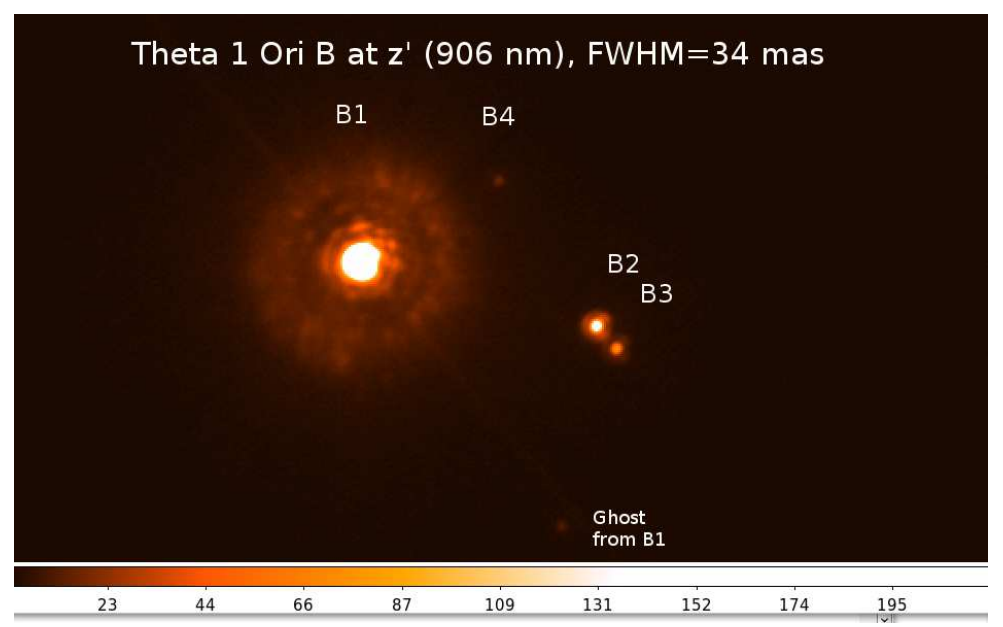

Fig. 9.- The MagAO z' $(0.91 \mu m)$ images of the $\theta^{1}$ Ori B group. Resolution 0.034". Linear color scale. North is up and east is left. Note that this image is $\sim 2 x$ sharper than that of the $8.4 \mathrm{~m} \mathrm{LBT}$ at $B r \gamma(2.16 \mu \mathrm{m})$ (see fig 8). This is clear evidence that AO in the "blue" allows a smaller $6.5 \mathrm{~m}$ telescope to outperform the resolution of an $8 \mathrm{~m}$ in the NIR. 


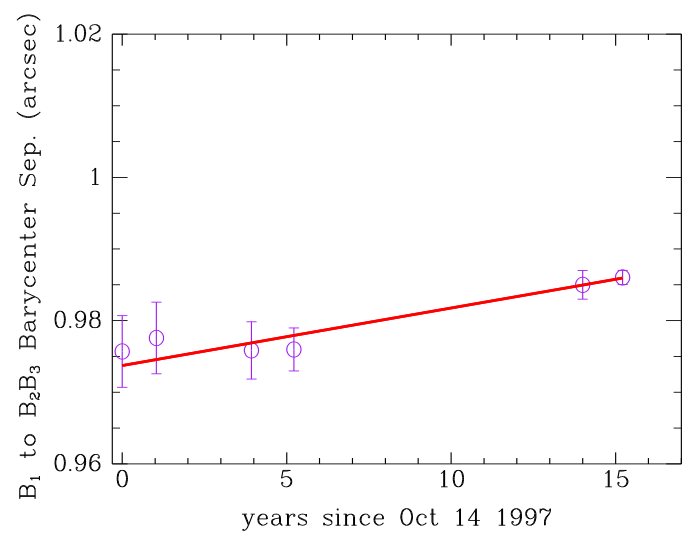

Fig. 10.- The separation between $\theta^{1}$ Ori $B_{1}$ and the barycenter of the $B_{2} B_{3}$ binary. Note how over 15 years of observation there has been a small, yet significant, relative proper motion observed $(0.80 \pm 0.18 \mathrm{mas} / \mathrm{yr}$; which is a significant correlation at the $97.4 \%$ level $)$. The first 2 data points are speckle observations from the 6-m SAO telescope (Weigelt et al. 1999), the next point is from Gemini/Hokupa'a observations Close et al. (2003c) followed by MMT AO observations Close et al. (2003c), and then LBT Close et al. (2012b), and the last from the MagAO system (this study).

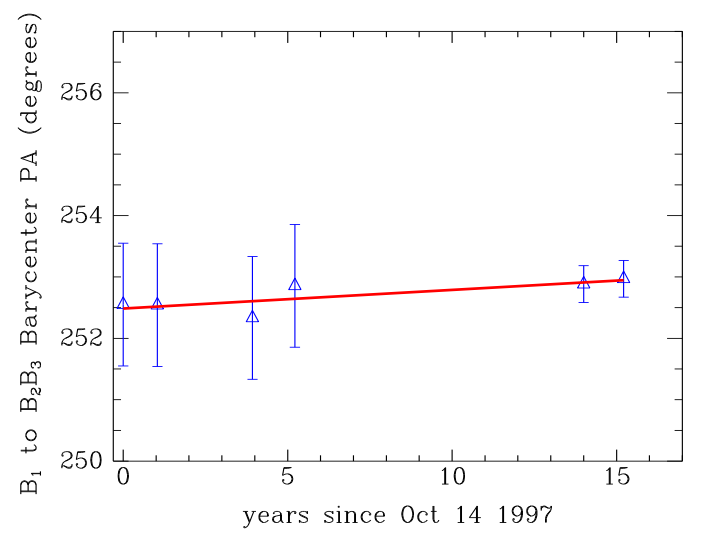

Fig. 11. - The position angle between $\theta^{1}$ Ori $B_{1}$ and the barycenter of the $B_{2} B_{3}$ binary. Note how over 15 years of observation there has been little relative PA motion observed $\left(0.030 \pm 0.044^{\circ} / \mathrm{yr}\right.$ which is just significant at the $88 \%$ level $)$. The epochs of the data are the same as in Fig. 10, 


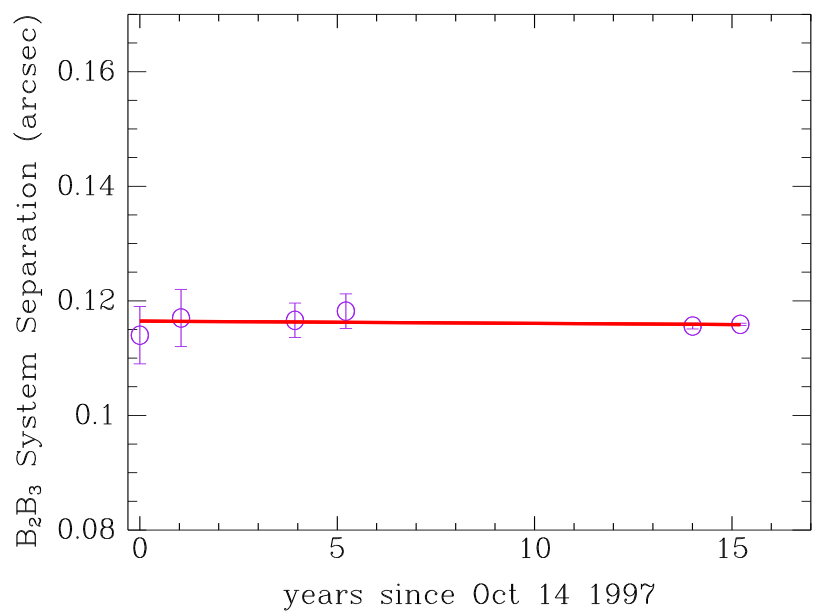

Fig. 12.- The separation between the $\theta^{1}$ Ori $B_{2}$ and $B_{3}$ components. Note the surprising lack of any significant relative motion $(-0.04 \pm 0.14$ mas $/ y r)$. The rms scatter from a constant value is only $0.14 \mathrm{mas} / \mathrm{yr}$. There appears to be very little change in the separation of the $B_{2} B_{3}$ system. The epochs of the data are the same as in Fig. 10,

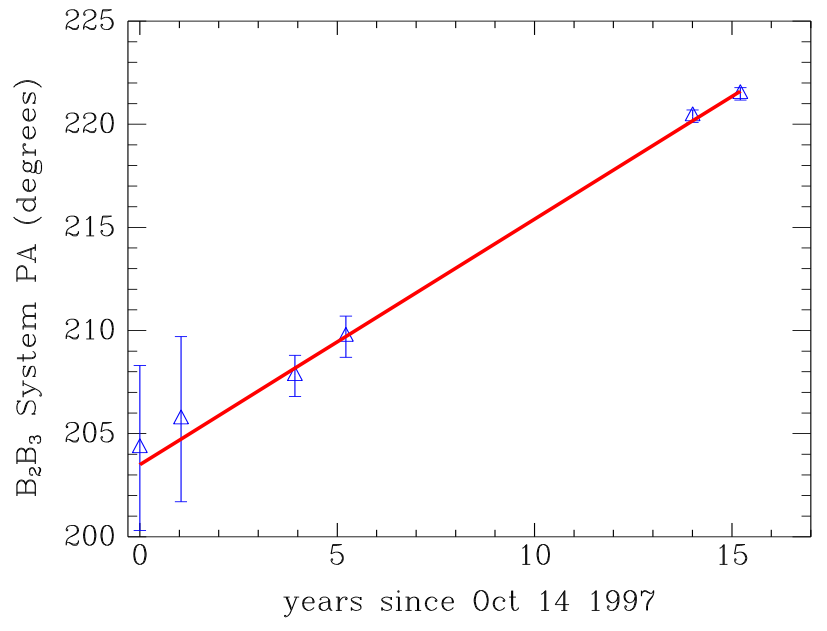

Fig. 13. - The position angle of $\theta^{1}$ Ori $B_{2}$ and $B_{3}$. Here we observe, clearly, real orbital arc of motion where $B_{3}$ moving counter-clockwise (at $1.19 \pm 0.06^{\circ} / \mathrm{yr}$; a correlation significant at the $99.9 \%$ level) around $B_{2}$. The epochs of the data are the same as in Fig. 10. 


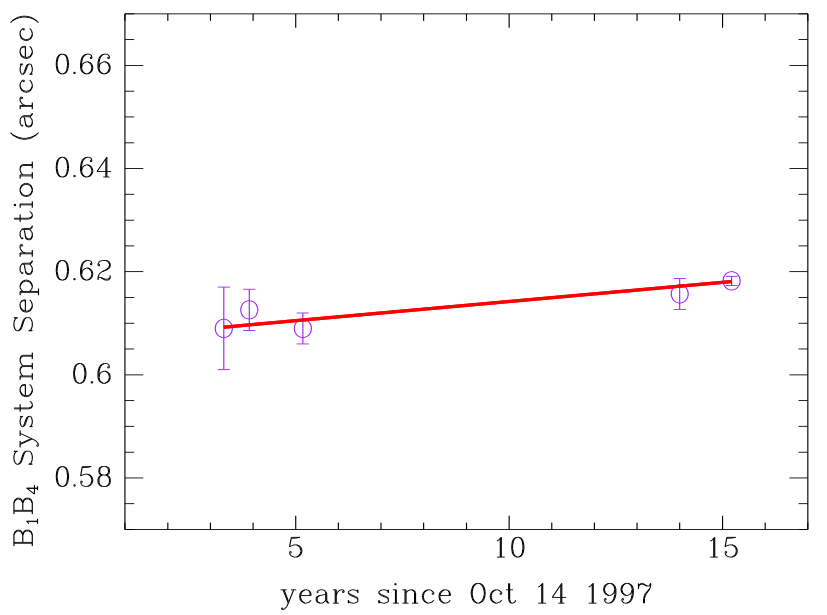

Fig. 14. - The separation between $\theta^{1}$ Ori $B_{1}$ and $B_{4}$. Note how over 13 years of observation there is little change in the separation $(0.72 \pm 0.23 \mathrm{mas} / \mathrm{yr}$; correlation $95 \%)$. The first data point is an speckle observation from the 6-m SAO telescope (Schertl et al. 2003), then Gemini/Hokupa'a observation Close et al. (2003c), the next data point is from the MMT AO observation Close et al. (2003c), the next from the LBT Close et al. (2012b), and the last is from MagAO.

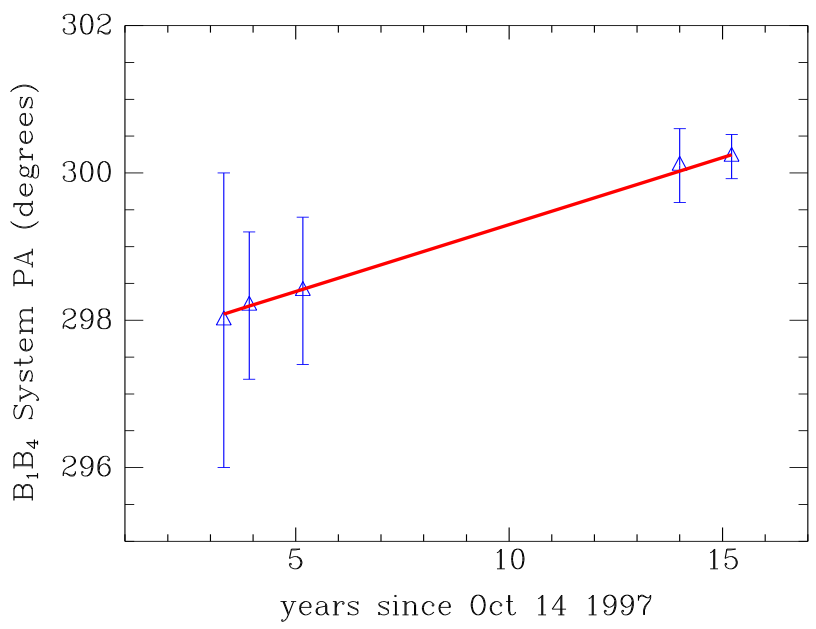

Fig. 15. - The position angle between $\theta^{1}$ Ori $B_{1}$ and $B_{4}$. Note how over 13 years of observation there has been only now clear significant relative proper motion observed $\left(0.181 \pm 0.067^{\circ} / \mathrm{yr}\right.$; correlation $\left.99.8 \%\right)$. The sources of the data is the same as in Fig. 14. 


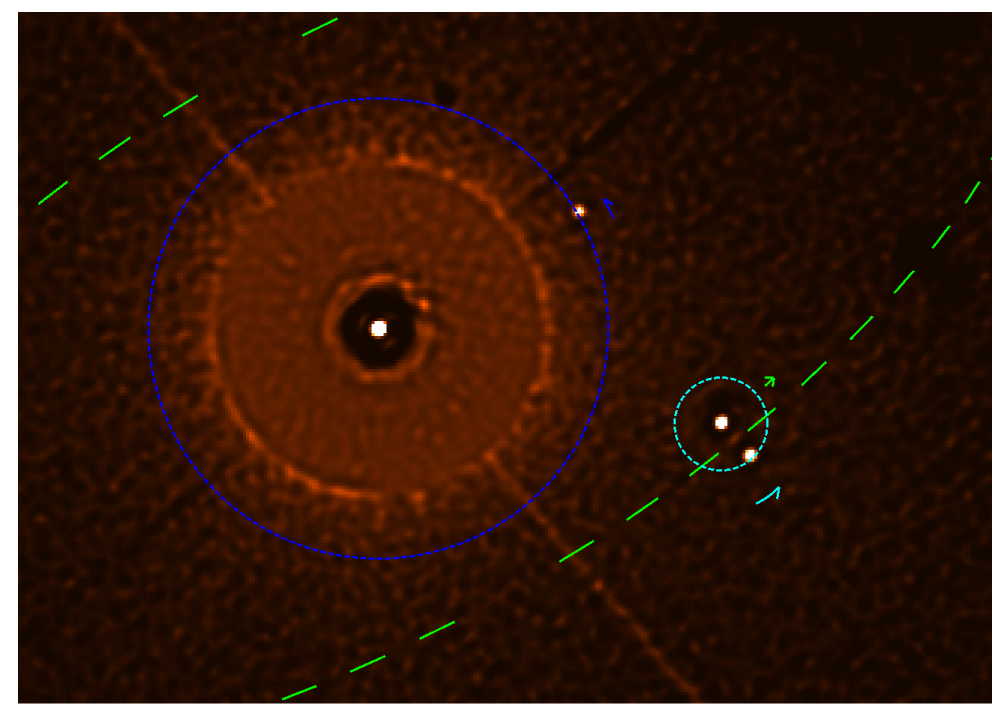

Fig. 16. - A possible model of the motions of the $\theta^{1}$ Ori $B$ group. Here we show a lucy deconvolved image of the $z^{\prime}$ image. We make an assumption that all the orbits are circular and plot possible orbital solutions for each component's orbit about $B_{1}$ based on this rough assumption. We also plot the actual observed orbital "arcs" so far imaged over the last 15 years for the system. Clearly the orbits are still undefined, but this plot gives some insight into the nature of the system.

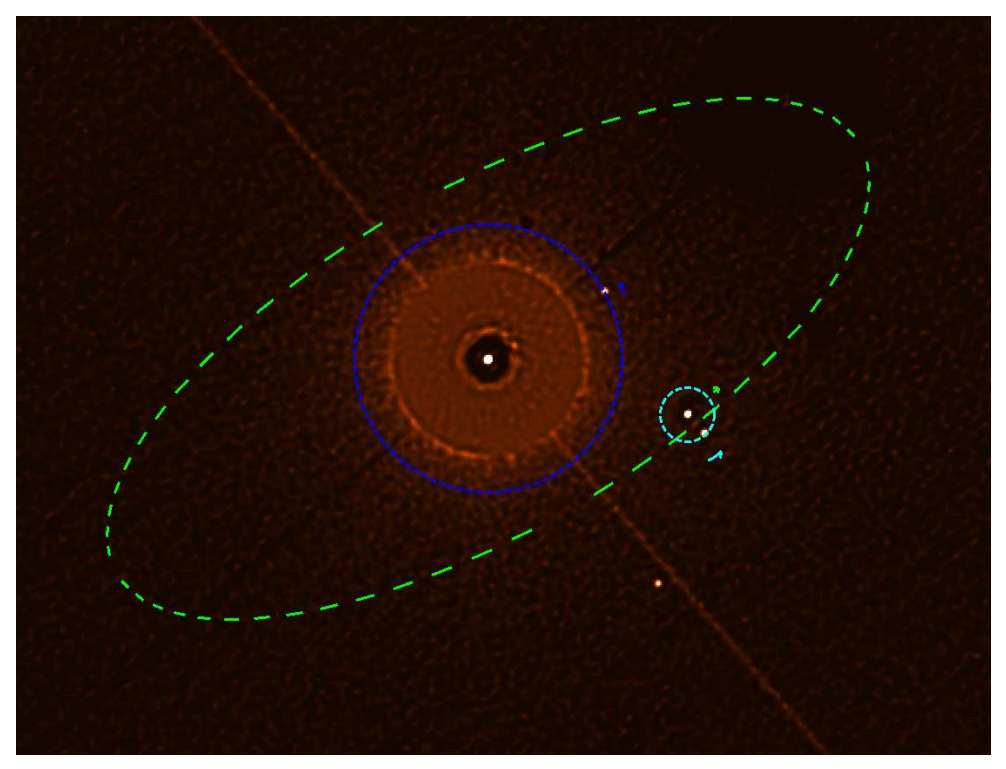

Fig. 17. - A zoom out of Fig. 16 to show the full inclined orbit of the $B_{2} B_{3}$ barycenter around $B_{1}$. 